\title{
Large-scale calculations of excitation energies in coupled cluster theory: The singlet excited states of benzene
}

\author{
Ove Christiansen, Henrik Koch, Asger Halkier, and Poul Jørgensen \\ Department of Chemistry, University of Aarhus, DK-8000 Aarhus C, Denmark \\ Trygve Helgaker \\ Department of Chemistry, University of Oslo, P.O. Box 1033 Blindern, N-0315 Oslo, Norway \\ Alfredo Sánchez de Merás \\ Department of Physical Chemistry, Valencia University, E-46100 Burjassot, Spain
}

(Received 22 May 1996; accepted 1 July 1996)

\begin{abstract}
Algorithms for calculating singlet excitation energies in the coupled cluster singles and doubles (CCSD) model are discussed and an implementation of an atomic-integral direct algorithm is presented. Each excitation energy is calculated at a cost comparable to that of the CCSD ground-state energy. Singlet excitation energies are calculated for benzene using up to 432 basis functions. Basis-set effects of the order of $0.2 \mathrm{eV}$ are observed when the basis is increased from augmented polarized valence double-zeta (aug-cc-pVDZ) to augmented polarized valence triple-zeta (aug-cc-pVTZ) quality. The correlation problem is examined by performing calculations in the hierarchy of coupled cluster models CCS, CC2, CCSD, and CC3, as well as by using the CCSDR(3) perturbative triples corrections. The effect of triple excitations are less than $0.2 \mathrm{eV}$ for all excitations except for the $2{ }^{1} E_{2 g}$ state. The calculated excitation energies are compared with experiment and other theoretical results. () 1996 American Institute of Physics. [S0021-9606(96)01038-0]
\end{abstract}

\section{INTRODUCTION}

The accurate calculation of electronic excitation energies remains a difficult challenge to theoretical chemistry since a balanced description of two electronic states is required. The methods used for the calculation of excitation energies may be divided in two classes: 1) methods that require the explicit calculation of individual states followed by the evaluation of the excitation energy as an energy difference, and (2) the response-function methods where the excitation energy is obtained directly from an eigenvalue equation. The first class of methods are exemplified by multireference configuration interaction (MRCI) (Ref. 1) and multireference perturbation theory (MRMP). ${ }^{2-4}$ The response-function methods are exemplified by self-consistent field (SCF) response theory, ${ }^{5}$ the second-order polarization-propagator approximation (SOPPA) ${ }^{6-7}$ and coupled cluster (CC) response theory. ${ }^{8-15}$

In response-function theory, the excitation energies are identified as the poles of the linear response function and the transition moments are obtained from their residues. In this approach, the excitation energies are obtained as the eigenvalues of the linear-response eigenvalue equations. ${ }^{5}$ Excitation energies and oscillator strengths are determined from the same response function and are thus obtained at the same level of approximation. Response functions can also be derived within coupled cluster theory. Although the ground state is obtained by solving a non-linear set of equations, response theory leads to a set of eigenvalue equations for the excited states, in contrast to for example the non-linear optimization of the individual states performed in calculations employing second-order perturbation theory building on a complete active space reference (CASPT2). ${ }^{3,4}$
A great advantage of the single-reference coupled cluster response method is that it provides us with a black-box approach to the calculation excitation energies. The calculation is completely specified when the orbital basis and the coupled cluster model-for example, the coupled cluster singles and doubles (CCSD) model-have been specified. Thus CCSD is defined universally a priori, making the calculations easier and enhancing the reliability of transferring error estimates between different molecules. In contrast, the CASPT2 calculation is not specified until the active spaces of the individual states have been selected-the active spaces are characteristic of each electronic state for each molecule and their construction requires a careful consideration of the state in question.

The quality of excitation energies obtained within the framework of coupled cluster response theory depends on two crucial factors: the adequacy of a single-determinant reference function in the coupled cluster ground-state calculation, and the adequacy of the excitation manifold for describing the excited states. To satisfy the first requirement, the Hartree-Fock wave function must provide a reasonable approximation for the ground state. This requirement therefore restricts the application of coupled cluster response theory to electronic systems that are dominated by a single determinant in the electronic ground state. Passing to the second requirement for the accurate calculation of excitation energies, we note that transitions dominated by a single electronic replacement are more accurately described than those dominated by a double replacement. ${ }^{14,15}$

In state-specific approaches such as CASPT2, the quality of the calculations do not depend strongly on the excitation level but are instead dependent on the multiconfigurational 
nature of the excited state. CASPT2 and single-reference CC response theory are therefore in some sense complementary. Single-reference CC response theory may be carried out in a black-box manner, providing the user with oscillator strengths and excitations energies calculated from the same model. CASPT2 requires more intervention on the part of the user but can be applied to multiconfigurational ground states. However, the separate treatment of non-dynamical and dynamical correlation in CASPT2 may in some cases give problems associated with intruder states. ${ }^{2}$ In benchmark calculations, the accuracy of CASPT2 ground-state electronic energies was found to be no higher than that of second-order Møller-Plesset theory (MP2) with regard to dynamical correlation., ${ }^{3,46}$ To obtain accurate excitation energies in CASPT2, it is thus crucial to have a balanced description of the two states and considerable care must be exercised in choosing their reference spaces. The non-linear simultaneous optimization of several electronic states is difficult and stateaverage approaches are sometimes used. Since the excited states are individually optimized in CASSCF, they are nonorthogonal and interacting. Special consideration is therefore required to obtain oscillator strengths in CASSCF for separately optimized states. ${ }^{17}$ Second-order corrections to the transition moments compatible with CASPT2 have so far not been considered, although CASPT2 energy differences are often used in the expression for the oscillator strengths ${ }^{2}$ where the transition moments are calculated from CAS functions. ${ }^{17}$

The coupled cluster approach has since its introduction in quantum chemistry gained increasing popularity, providing an efficient method for treating the dynamical correlation as evident from many recent applications. ${ }^{18-20}$ Several different implementations of the CCSD model have been presented $^{21-27}$ and a variety of molecular properties have been calculated-molecular gradients ${ }^{28-30}$ and molecular Hessians, ${ }^{31}$ polarizabilities, ${ }^{32-34}$ nuclear magnetic shielding constants, ${ }^{35-38}$ as well as other spectroscopic constants. ${ }^{39,40,41}$ The $\operatorname{CCSD}(\mathrm{T})$ approach $^{42}$ - which includes perturbative corrections for the effect of triple excitations-has provided highly accurate results for a wide range of frequency-independent molecular properties and remains the most popular method for high-accuracy calculations. For the calculation of time-independent properties, the sequence of models SCF, MP2, CCSD, and CCSD(T) provides a useful hierarchy of methods where the properties may be calculated to higher and higher accuracy at increasing cost.

In a recent series of papers, we have advanced a new hierarchy of coupled cluster models. The standard hierarchy of coupled cluster models CCS, CCSD, CCSDT, and so on is supplemented with the iterative models CC2 (Ref. 15) and CC3 (Refs. 43, 14) introduced as approximations to CCSD and CCSDT (and similarly for higher orders). The advantage of this new hierarchy is that we may to each order identify excitation energies and transition moments from the response functions. As such, the $\mathrm{CC} 2$ and $\mathrm{CC} 3$ models provide useful alternatives to the perturbative MP2 and $\operatorname{CCSD}(\mathrm{T})$ models, which do not give response functions that possess pole structures in accordance with the exact theory and thus cannot be applied successfully to the study of dynamic properties, as is for example evident from inspection of the expression for the MP2 frequency-dependent polarizabilities. ${ }^{44,45}$

The new hierarchy of coupled cluster methods contains the models $\operatorname{CCS}\left(N^{4}\right), \operatorname{CC} 2\left(N^{5}\right), \operatorname{CCSD}\left(N^{6}\right), \operatorname{CC} 3\left(N^{7}\right)$, $\operatorname{CCSDT}\left(N^{8}\right)$, and so on, where the numbers in parentheses indicate the computational cost in terms of the scaling of the calculations with the number of orbitals $N$. Going through the sequence of models belonging to this hierarchy, we may calculate molecular dynamic properties with increasing accuracy and cost. We have examined the performance of the above coupled cluster hierarchy in benchmark calculations of excitation energies. ${ }^{46,47}$ The inherent convergence-in terms of increasing completeness of the cluster expansion and also the determination of properties through increasing order in the ground-state fluctuation potential-manifests itself clearly through a decrease in error of about a factor of 3 at each step.

Iterative triples methods are computationally expensive and we have therefore proposed non-iterative corrections to CCSD. ${ }^{48}$ In particular, we have introduced the CCSDR(3) approach as a non-iterative approach for the calculation of excitation energies that includes the same lower-order terms as CC3. The CCSDR(3) approach was found to give highly accurate excitation energies ${ }^{48,47}$ and to be superior to other non-iterative triples corrections. ${ }^{48-49}$

A hierarchical approach is difficult to obtain in multireference methods-extensions of the active space cannot always be expected to give better excitation energies and the convergence towards the exact result in configuration interaction is slow. Also, perturbation theory cannot always be relied on to improve the description systematically. It has been demonstrated that M $\phi$ ller-Plesset theory for several single-reference systems such as $\mathrm{Ne}$ is divergent for extended basis sets. ${ }^{86}$ For multireference systems, an even more unpredictable behaviour is found ${ }^{87}$

Atomic-integral direct methods were introduced by Almlöf and coworkers ${ }^{50}$ and have significantly extended the application range of the Hartree-Fock and MP2 methods ${ }^{88,89}$ A similar development has recently taken place in CC theory, where atomic-integral direct CCSD techniques have been presented based on a strategy where integrals are generated in distributions with one fixed and three free atomic indices. ${ }^{26,27}$ Thus, ground-state CCSD total energies have been calculated with more than 500 basis functions. ${ }^{27}$

We present in this paper the extension of such techniques to the calculation of CCSD excitation energies. We have briefly described elsewhere the techniques for integraldirect calculations of $\mathrm{CC} 2$ excitation energies. ${ }^{51}$ The linear transformations that are essential for integral-direct CC calculations of excitation energies are also required for the calculation of other molecular properties. In this paper, we discuss algorithms for calculating the linear transformations necessary to calculate CCSD excitation energies and eigenvectors. We describe in detail the integral-direct implementation and especially the connection to the integral-direct algorithm for the ground-state energy described in Ref. 27.

We present in this paper applications to the singlet ex- 
cited states in the benzene molecule. Benzene is one of the most investigated molecules both theoretically ${ }^{52-60}$ and experimentally. ${ }^{60-73,79-82}$ However, there are still many unsolved problems in the benzene spectrum. The application of integral-direct techniques significantly expands the range of one electron basis sets that can be used in theoretical calculations. The recently presented CC2 calculations and the calculations in this paper represent to our knowledge the first integral-direct correlated calculations of excitation energies. We here present calculations with up to 432 basis functions in CCSD. The effect of triple excitations is investigated by carrying out $\mathrm{CC} 3$ and $\operatorname{CCSDR}(3)$ calculations.

In Section II, we review the theory for calculating coupled cluster excitation energies and describe an implementation using an integral-direct algorithm. In Section III, we present results for benzene and compare our results with other theoretical and experimental results. In Section IV we give a summary.

\section{EXCITATION ENERGIES IN THE INTEGRAL-DIRECT CCSD MODEL}

\section{A. Coupled-cluster theory}

Consider a closed-shell system described by a Hamiltonian $\mathrm{H}$. The single-reference coupled cluster (CC) ansatz for the wave function is

$$
|\mathrm{CC}\rangle=\exp (T)|H F\rangle,
$$

where the reference state is taken to be a Hartree-Fock $|H F\rangle$ state. For an $N$-electron system, the cluster operator in Eq. (1) truncates at excitation level $N$

$$
T=T_{1}+T_{2}+\cdots+T_{N},
$$

where

$$
\begin{aligned}
& T_{1}=\sum_{a i} t_{i}^{a} E_{a i}, \\
& T_{2}=\sum_{(a i) \geqslant(b j)} t_{i j}^{a b} E_{a i} E_{b j},
\end{aligned}
$$

are the one- and two-electron cluster operators and similarly for higher-order excitation operators. Indices $i j k l$ and $a b c d$ refer to the occupied and unoccupied orbitals in the HartreeFock reference state $|H F\rangle$. In a shorthand notation, we write the cluster operator in the form

$$
T=\sum_{\mu} t_{\mu} \tau_{\mu}
$$

where the $t_{\mu}$ are the cluster amplitudes and $\tau_{\mu}$ the corresponding excitation operators. Introducing the $\mathrm{CC}$ ansatz into the Schrödinger equation and multiplying with $\exp (-T)$ from the left, we arrive at the CC Schrödinger equation

$$
\exp (-T) H \exp (T)|H F\rangle=E|H F\rangle .
$$

The cluster amplitudes are determined by projecting the $\mathrm{CC}$ Schrödinger equation onto the manifold of excitations out of the reference state

$$
\langle\mu|=\langle H F| \tau_{\mu}^{+},
$$

which gives the $\mathrm{CC}$ amplitude equations

$$
\Omega_{\mu}=\langle\mu|\exp (-T) H| \mathrm{CC}\rangle=0 .
$$

Projection of the CC Schrödinger equation onto the reference state gives the $\mathrm{CC}$ energy as

$$
E=\langle H F|H| \mathrm{CC}\rangle \text {. }
$$

Several derivations of coupled cluster response functions have been presented. ${ }^{8-15}$ The excitation energies are identified as poles of the linear response function, which determines the excitation energies as the eigenvalues of the nonsymmetric coupled-cluster Jacobian

$$
\mathbf{A} \mathbf{R}_{k}=\omega_{k} \mathbf{R}_{k}
$$

We here assume a unit metric

$$
S_{\mu \nu}=\left\langle\mu\left|\exp (-T) \tau_{\nu}\right| \mathrm{CC}\right\rangle=\delta_{\mu \nu} .
$$

The coupled cluster Jacobian is defined as

$$
A_{\mu \nu}=\frac{\partial \Omega_{\mu}}{\partial t \nu} .
$$

For a non-approximated CC theory, we obtain from Eq. (8)

$$
A_{\mu \nu}=\left\langle\mu\left|\exp (-T)\left[H, \tau_{\nu}\right]\right| \mathrm{CC}\right\rangle .
$$

In the Lagrangian pseudo-energy derivative formulation of $\mathrm{CC}$ response theory in Ref. 14, the excitation energies in any iterative coupled cluster model are determined as eigenvalues of the Jacobian defined in Eq. (12). This holds not only when the cluster expansion is truncated as in CCSD, but also when additional approximations are introduced as for example in CC2 and CC3.

\section{B. The coupled cluster singles and doubles model}

In the coupled cluster singles and doubles model (CCSD), the cluster expansion is truncated at the doubles excitation level

$$
T=T_{1}+T_{2} \text {. }
$$

The CCSD amplitudes $\left\{t_{i}^{a}\right\}$ and $\left\{t_{i j}^{a b}\right\}$ are determined from Eq. (8), where $\{<\mu \mid\}$ are singly and doubly excited determinants with respect to the Hartree-Fock reference determinant

$$
\begin{aligned}
\left\{\left\langle\mu_{1}\right|\right\}= & \left\{\langle H F| E_{i a} \frac{1}{2}\right\}, \\
\left\{\left\langle\mu_{2}\right|\right\}= & \left\{\langle H F|\left(2 E_{i a} E_{j b}+E_{j a} E_{i b}\right)\right. \\
& \left.\times \frac{1}{6}\left(1+\delta_{a b} \delta_{i j}\right)^{-1} \mid a i \geqslant b j\right\} .
\end{aligned}
$$

Together with the excited states $E_{a i}|H F\rangle$ and $E_{a i} E_{b j}|H F\rangle$ obtained using the excitation operators in Eqs. (3) and (4), these states constitute a biorthonormal basis.

Following Ref. 26, the CCSD amplitude equations can be expressed as 


$$
\begin{aligned}
\Omega_{\mu} & =\left\langle\mu\left|\exp \left(-T_{1}-T_{2}\right) H \exp \left(T_{1}+T_{2}\right)\right| H F\right\rangle \\
& =\left\langle\mu\left|\exp \left(-T_{2}\right) \hat{H} \exp \left(T_{2}\right)\right| H F\right\rangle=0,
\end{aligned}
$$

where we have introduced the $T_{1}$-similarity transformed Hamiltonian

$$
\hat{H}=\exp \left(-T_{1}\right) H \exp \left(T_{1}\right) .
$$

The electronic Hamiltonian in the second-quantization formalism is given by

$$
H=\sum_{p q} h_{p q} E_{p q}+\frac{1}{2} \sum_{p q r s}(p q \mid r s) e_{p q r s} .
$$

The molecular orbitals $\left\{\phi_{p}\right\}$ are expanded in the atomic orbitals $\left\{\chi_{\mu}\right\}$

$$
\phi_{p}=\sum_{\mu} C_{\mu p} \chi_{\mu},
$$

where $C_{\mu p}$ are the molecular-orbital coefficients. Since $T_{1}$ is a one-particle operator, the transformation in Eq. (18) conserves the particle rank of the electronic Hamiltonian. The $T_{1}$ transformation of the Hamiltonian can be expressed in terms of a transformation of the creation and annihilation operators. Absorbing this transformation in the definition of the integrals, we may write the modified Hamiltonian in Eq. (18) as

$$
\hat{H}=\hat{h}+\hat{g}=\sum_{p q} \hat{h}_{p q} E_{p q}+\frac{1}{2} \sum_{p q r s}(p q \hat{\mid} r s) e_{p q r s} .
$$

The integrals of the modified Hamiltonian are

$$
\begin{aligned}
& \hat{h}_{p q}=\sum_{\mu \nu} \Lambda_{\mu p}^{p} \Lambda_{\nu q}^{h} h_{\mu \nu}, \\
& (p q \mid \hat{\mid} r s)=\sum_{\substack{\mu \nu \\
\rho \sigma}} \Lambda_{\mu p}^{p} \Lambda_{\rho r}^{p} \Lambda_{\nu q}^{h} \Lambda_{\sigma s}^{h}(\mu \nu \mid \rho \sigma),
\end{aligned}
$$

where $\Lambda^{p}$ and $\Lambda^{h}$ are effective MO transformation matrices (particle and hole transformations) given in terms of molecular orbital coefficients and the singles amplitudes

$$
\begin{aligned}
& \Lambda^{p}=C\left[1-t_{1}^{T}\right], \\
& \Lambda^{h}=C\left[1+t_{1}\right] .
\end{aligned}
$$

We have here introduced the matrix notation

$$
t_{1}=\left\{\begin{array}{cc}
0 & 0 \\
\left\{t_{a i}\right\} & 0
\end{array}\right\}
$$

where the orbitals are ordered with the occupied orbitals preceding the unoccupied ones. The usual eightfold permutational symmetry of the two-electron integrals in the electronic Hamiltonian is destroyed and only the particle permutation symmetry is conserved

$$
(p q \hat{\mid} r s)=(r s \hat{\mid} p q) \text {. }
$$

Using the $T_{1}$-transformed Hamiltonian, the CCSD amplitude equations can be written in a coupled cluster doubles (CCD) form:

$$
\begin{aligned}
& \left\langle\mu_{1}\left|\hat{H}+\left[\hat{H}, T_{2}\right]\right| H F\right\rangle=0, \\
& \left\langle\mu_{2}\left|\hat{H}+\left[\hat{H}, T_{2}\right]+\frac{1}{2}\left[\left[\hat{H}, T_{2}\right], T_{2}\right]\right| H F\right\rangle=0 .
\end{aligned}
$$

The CCSD Jacobian is given by

$$
A_{\mu \nu}=\left\langle\mu\left|\exp \left(-T_{1}-T_{2}\right)\left[H, \tau_{\nu}\right] \exp \left(T_{1}+T_{2}\right)\right| H F\right\rangle .
$$

The manipulations applied to the CCSD amplitude equations above are easily introduced in the CCSD Jacobian:

$$
\begin{aligned}
A_{\mu \nu} & =\left\langle\mu\left|\exp \left(-T_{2}\right)\left[\hat{H}, \tau_{\nu}\right] \exp \left(T_{2}\right)\right| H F\right\rangle \\
& =\left\langle\mu\left|\left[\hat{H}, \tau_{\nu}\right]\right| H F\right\rangle+\left\langle\mu\left|\left[\left[\hat{H}, \tau_{\nu}\right], T_{2}\right]\right| H F\right\rangle .
\end{aligned}
$$

We may now write the CCSD Jacobian in matrix form as

$$
A_{\mu \nu}=\left\{\begin{array}{cc}
\left\langle\mu_{1}\left|\left[\hat{H}+\left[\hat{H}, T_{2}\right], \tau_{\nu_{1}}\right]\right| H F\right\rangle & \left\langle\mu_{1}\left|\left[\hat{H}, \tau_{\nu_{2}}\right]\right| H F\right\rangle \\
\left\langle\mu_{2}\left|\left[\hat{H}+\left[\hat{H}, T_{2}\right], \tau_{\nu_{1}}\right]\right| H F\right\rangle & \left\langle\mu_{2}\left|\left[\hat{H}+\left[H, T_{2}\right], \tau_{\nu_{2}}\right]\right| H F\right\rangle
\end{array}\right\} .
$$

\section{Transformation of trial vectors with the CCSD Jacobian}

In solving large eigenvalue equations, iterative techniques are mandatory. The key computational step in iterative techniques is the linear transformation of a trial vector with a matrix. To extend the integral-direct coupled cluster technique to the calculation of $\mathrm{CC}$ excitation energies and second-order molecular properties, it is therefore necessary to perform linear transformations with the CC Jacobian in an AO-integral driven approach.

Let $\mathbf{R}$ denote a right trial vector with singles and doubles excitation components $\mathbf{R}_{1}$ and $\mathbf{R}_{2}$, respectively. The transformed vector may be written

$$
\boldsymbol{\rho}=\mathbf{A R}
$$

or in terms of the singles and doubles excitation components

$$
\left(\begin{array}{l}
\boldsymbol{\rho}_{1} \\
\boldsymbol{\rho}_{2}
\end{array}\right)=\left(\begin{array}{cc}
{ }^{1} \boldsymbol{\rho}_{1}+{ }^{2} \boldsymbol{\rho}_{1} \\
{ }^{1} \boldsymbol{\rho}_{2}+{ }^{2} \boldsymbol{\rho}_{2}
\end{array}\right)=\left(\begin{array}{c}
\mathbf{A}_{11} \mathbf{R}_{1}+\mathbf{A}_{12} \mathbf{R}_{2} \\
\mathbf{A}_{21} \mathbf{R}_{1}+\mathbf{A}_{22} \mathbf{R}_{2}
\end{array}\right) .
$$

Introducing the CCSD Jacobian in the form of Eq. (32), we obtain 


$$
\begin{aligned}
& { }^{1} \rho_{a i}=\left\langle\begin{array}{c}
a \\
i
\end{array}\left|\left[\hat{H}, R_{1}\right]\right| H F\right\rangle+\left\langle\begin{array}{c}
a \\
i
\end{array}\left|\left[\left[\hat{H}, R_{1}\right], T_{2}\right]\right| H F\right\rangle, \\
& { }^{1} \rho_{a i b j}=\left\langle\begin{array}{c}
a b \\
i j
\end{array}\left|\left[\hat{H}, R_{1}\right]\right| H F\right\rangle+\left\langle\begin{array}{r}
a b \\
i j
\end{array}\left|\left[\left[\hat{H}, R_{1}\right], T_{2}\right]\right| H F\right\rangle, \\
& { }^{2} \rho_{a i}=\left\langle\begin{array}{c}
a \\
i
\end{array}\left|\left[\hat{H}, R_{2}\right]\right| H F\right\rangle, \\
& { }^{2} \rho_{a i b j}=\left\langle\begin{array}{c}
a b \\
i j
\end{array}\left|\left[\hat{H}, R_{2}\right]\right| H F\right\rangle+\left\langle\begin{array}{r}
a b \\
i j
\end{array}\left|\left[\left[H, R_{2}\right], T_{2}\right]\right| H F\right\rangle,
\end{aligned}
$$

where $\left\langle{ }_{i}^{a}\right|$ and $\left\langle{ }_{i j}^{a b}\right|$ refer to the biorthonormal basis in Eqs. (15) and (16). $R_{1}$ and $R_{2}$ are singles and doubles excitation operators with the trial-vector coefficients $\left(R_{k}^{c}, R_{k l}^{c d}\right)$ as amplitudes. (We use the same letters for the operators and vectors but use bold-face type for the vectors.) Thus, the $R_{1}$ and $R_{2}$ operators are written in a form similar to the $T_{1}$ and $T_{2}$ operators

$$
\begin{aligned}
& R_{1}=\sum_{c k} R_{k}^{c} E_{c k}, \\
& R_{2}=\sum_{(c k) \geqslant(d l)} R_{k l}^{c d} E_{c k} E_{d l} .
\end{aligned}
$$

We introduce yet another effective Hamiltonian

$$
\widetilde{H}=\left[\hat{H}, R_{1}\right],
$$

in terms of which we may rewrite Eqs. (35) and (36) as

$$
\begin{aligned}
& { }^{1} \rho_{a i}=\left\langle\begin{array}{c}
a \\
i
\end{array}|\widetilde{H}| H F\right\rangle+\left\langle\begin{array}{c}
a \\
i
\end{array}\left|\left[\tilde{H}, T_{2}\right]\right| H F\right\rangle, \\
& { }^{1} \rho_{a i b j}=\left\langle\begin{array}{c}
a b \\
i j
\end{array}|\widetilde{H}| H F\right\rangle+\left\langle\begin{array}{c}
a b \\
i j
\end{array}\left|\left[\widetilde{H}, T_{2}\right]\right| H F\right\rangle .
\end{aligned}
$$

The effective Hamiltonian in Eq. (41) represents a one-index transformation of the $T_{1}$-transformed Hamiltonian. Consider the one-electron part:

$$
\begin{aligned}
\tilde{h} & =\left[\hat{h}, R_{1}\right] \\
& =\left[\sum_{m n} \hat{h}_{m n} E_{m n}, \sum_{a i} R_{i}^{a} E_{a i}\right] \\
& =\sum_{m n} \sum_{a i} \hat{h}_{m n} R_{i}^{a}\left(E_{m i} \delta_{n a}-E_{a n} \delta_{m i}\right) \\
& =\sum_{m i}\left(\sum_{a} \hat{h}_{m a} R_{i}^{a}\right) E_{m i}+\sum_{a n}\left(-\sum_{i} \hat{h}_{i n} R_{i}^{a}\right) E_{a n} \\
& =\sum_{m i} \hat{h}_{m} E_{i} E_{m i}+\sum_{a n} \hat{h}_{\bar{a} n} E_{a n} .
\end{aligned}
$$

An overbar indicates an additional transformation of that index with the $R_{1}$ amplitudes in accordance with the equation above. We may now write the $R_{1}$-effective Hamiltonian as

$$
\widetilde{H}=\sum_{p q} \widetilde{h}_{p q} E_{p q}+\frac{1}{2} \sum_{p q r s}(p q \widetilde{\Gamma} r s) e_{p q r s},
$$

where the tilde integrals are defined as

$$
\begin{aligned}
& \tilde{h}_{p q}=\hat{h}_{\overline{p q}}+\hat{h}_{p \bar{q}}, \\
& (p q \widetilde{\Gamma} r s)=(\overline{p q} \widetilde{\Gamma} r s)+(p \bar{q} \widetilde{\Gamma} r s)+(p q \widetilde{\Gamma} \bar{r} s)+(p q \widetilde{\Gamma} r \bar{s}) .
\end{aligned}
$$

Again, we may absorb these one-index transformations into the MO coefficients. Consider for example the case where the particle index of the one-electron operator is transformed

$$
\begin{aligned}
\hat{h}_{\bar{p} q} & =-\sum_{k} R_{k}^{p} \hat{h}_{k q}=\sum_{\mu \nu}\left(-\sum_{k} R_{k}^{p} \Lambda_{\mu k}^{p}\right) \Lambda_{\nu q}^{h} h_{\mu \nu} \\
& =\sum_{\mu \nu} \bar{\Lambda}_{\mu p}^{p} \Lambda_{\nu q}^{h} h_{\mu \nu} .
\end{aligned}
$$

We have here introduced $\bar{\Lambda}^{p}$ and $\bar{\Lambda}^{h}$ as new effective MO transformation matrices that incorporate the new $R_{1}$ transformation:

$$
\begin{aligned}
& \bar{\Lambda}_{\mu p}^{p}=-\sum_{k} R_{k}^{p} \Lambda_{\mu k}^{p}, \\
& \bar{\Lambda}_{\mu q}^{h}=\sum_{a} R_{q}^{a} \Lambda_{\mu a}^{h} .
\end{aligned}
$$

Note that the structures of $\bar{\Lambda}^{p}$ and $\bar{\Lambda}^{h}$ imply that a "barred", particle index must be virtual and that a "barred" hole index must be occupied, for example

$$
\hat{h}_{\bar{i} m}=0 \text { and } \hat{h}_{m \bar{a}}=0 \text {. }
$$

We may now compare the coupled cluster amplitude equations in form of the CCSD vector function in Eqs. (28) and (29) with the expressions for the linearly transformed vector in Eqs. (37), (38), (42), (43). The individual terms in the transformed vector are all present in the CCSD vector function. The terms in the CCSD amplitude equations that are linear in $T_{2}$ become linear in $R_{2}$. In place of terms quadratic in $T_{2}$ such as $1 / 2\left[\left[H, T_{2}\right], T_{2}\right]$ we obtain terms bilinear in $R_{2}$ and $T_{2}$, [ $\left.\left[H, T_{2}\right], R_{2}\right]$. In the $R_{1}$ terms Eqs. (42) and (43) we have absorbed $R_{1}$ into the Hamiltonian. As a consequence, we now have terms similar to the terms in Eqs. (28) and (29) where $\hat{H}$ is replaced with $\widetilde{H}$. Note, however, that the terms quadratic in $T_{2}$ vanish since $R_{1} T_{2}^{2}$ is a quintuple excitation. The CCSD amplitude equations in the biorthonormal basis can be written as follows

$$
\begin{aligned}
\Omega_{a i}= & \Omega_{a i}^{G}+\Omega_{a i}^{H}+\Omega_{a i}^{I}+\Omega_{a i}^{J}, \\
\Omega_{a i b j}= & \left(\Omega_{a i b j}^{A}+\Omega_{a i b j}^{B}+P_{i j}^{a b}\left\{\Omega_{a i b j}^{C}+\Omega_{a i b j}^{D}+\Omega_{a i b j}^{E}\right\}\right. \\
& \left.+\Omega_{a i b j}^{F}\right)\left(1+\delta_{i j} \delta_{a b}\right)^{-1},
\end{aligned}
$$

where

$$
P_{i j}^{a b}\left(\begin{array}{c}
a b \\
i j
\end{array}\right)=\left(\begin{array}{c}
a b \\
i j
\end{array}\right)+\left(\begin{array}{c}
b a \\
j i
\end{array}\right) .
$$

In a similar way, we may write the linearly transformed vector as

$$
{ }^{1} \rho_{a i}={ }^{1} \rho_{a i}^{G}+{ }^{1} \rho_{a i}^{H}+{ }^{1} \rho_{a i}^{I}+{ }^{1} \rho_{a i}^{J},
$$


TABLE I. The CCSD linear transformation and the CCSD amplitude equations.

\begin{tabular}{|c|c|c|}
\hline${ }^{1} \rho_{a i}^{G}=\sum_{c d k} t_{i k}^{c d} \widetilde{L_{k d a c}}$ & ${ }^{2} \rho_{a i}^{G}=\sum_{c d k} R_{i k}^{c d} \hat{L}_{k d a c}$ & $\Omega_{a i}^{G}=\sum_{c d k} t_{i k}^{c d} \hat{L}_{k d a c}$ \\
\hline${ }^{1} \rho_{a i}^{H}=-\sum_{d k l} t_{k l}^{a d} \widetilde{L}_{l d k i}$ & ${ }^{2} \rho_{a i}^{H}=-\sum_{d k l} R_{k l}^{a d} \hat{L}_{l d k i}$ & $\Omega_{a i}^{H}=-\sum_{d k l} t_{k l}^{a d} \hat{L}_{l d k i}$ \\
\hline $\begin{array}{l}{ }^{1} \rho_{a i}^{I}=\sum_{c k}\left(2 t_{i k}^{a c}-t_{i k}^{c a}\right) \widetilde{F}_{k c} \\
{ }^{1} \rho_{a i}^{J}=\widetilde{F}_{a i}\end{array}$ & ${ }^{2} \rho_{a i}^{I}=\sum_{c k}\left(2 R_{i k}^{a c}-R_{i k}^{c a}\right) \hat{F}_{k c}$ & $\begin{array}{l}\Omega_{a i}^{I}=\sum_{c k}\left(2 t_{i k}^{a c}-t_{i k}^{c a}\right) \hat{F}_{k c} \\
\Omega_{a i}^{J}=\hat{F}_{a i}\end{array}$ \\
\hline${ }^{1} \rho_{a i b j}^{A}=\sum_{k l} t_{k l}^{a b}(k i \widetilde{\mid l j})$ & ${ }^{2} \rho_{a i b j}^{A}=\sum_{k l} R_{k l}^{a b}\left((k i \hat{\mid} l j)+\sum_{c d} t_{i j}^{c d}(k c \mid l d)\right)$ & $\Omega_{a i b j}^{A}=\sum_{k l} t_{k l}^{a b}\left((k i \hat{\mid} \mid j)+\sum_{c d} t_{i j}^{c d}(k c \mid l d)\right)$ \\
\hline${ }^{1} \rho_{a i b j}^{C}=-\left(\frac{1}{2}+P_{i j}\right) \sum_{c k} t_{j k}^{c b}(k i \widetilde{\mid a c})$ & ${ }^{2} \rho_{a i b j}^{C}=-\left(\frac{1}{2}+P_{i j}\right) \sum_{c k} R_{j k}^{c b}\left((k i \hat{\mid} a c)-\sum_{d l} t_{l i}^{a d}(l c \mid k d)\right)$ & $\Omega_{a i b j}^{C}=-\left(\frac{1}{2}+P_{i j}\right) \sum_{c k} t_{j k}^{c b}\left((k i \mid \hat{\mid a c})-\frac{1}{2} \sum_{d l} t_{l i}^{a d}(l c \mid k d)\right)$ \\
\hline${ }^{1} \rho_{a i b j}^{D}=\frac{1}{2} \sum_{c k}\left(2 t_{j k}^{b c}-t_{j k}^{c b}\right) \widetilde{L_{k c a i}}$ & ${ }^{2} \rho_{a i b j}^{D}=\frac{1}{2} \sum_{c k}\left(2 R_{j k}^{b c}-R_{j k}^{c b}\right)\left(\hat{L}_{k c a i}+\sum_{d l}\left(2 t_{i l}^{a d}-t_{i l}^{d a}\right) L_{l d k c}\right.$ & $\Omega_{a i b j}^{D}=\frac{1}{2} \sum_{c k}\left(2 t_{j k}^{b c}-t_{j k}^{c b}\right)\left(\hat{L}_{k c a i}+\frac{1}{2} \sum_{d l}\left(2 t_{i l}^{a d}-t_{i l}^{d a}\right) L_{l d k c}\right.$ \\
\hline${ }^{1} \rho_{a i b j}^{E 1}=\sum_{c} t_{i j}^{a c} \widetilde{F}_{b c}$ & ${ }^{2} \rho_{a i b j}^{E 1}=\sum_{c} R_{i j}^{a c}\left(\hat{F}_{b c}-\sum_{d l m} t_{l m}^{d b} L_{l d m c}\right)$ & $\Omega_{a i b j}^{E 1}=\sum_{c} t_{i j}^{a c}\left(\hat{F}_{b c}-\sum_{d l m} t_{l m}^{d b} L_{l d m c}\right)$ \\
\hline${ }^{1} \rho_{a i b j}^{E 2}=-\sum_{k} t_{i k}^{a c} \widetilde{F}_{k j}$ & ${ }^{2} \rho_{a i b j}^{E 2}=-\sum_{k} R_{i k}^{a b}\left(\hat{F}_{k j}+\sum_{d e m} t_{j m}^{d e} L_{m e k d}\right)$ & $\Omega_{a i b j}^{E 2}=-\sum_{k} t_{i k}^{a b}\left(\hat{F}_{k j}+\sum_{d e m} t_{j m}^{d e} L_{m e k d}\right)$ \\
\hline${ }^{1} \rho_{a i b j}^{F}=(a \widetilde{i \mid b j})$ & & $\Omega_{a i b j}^{F}=(a i \hat{\mid} \mid b j)$ \\
\hline
\end{tabular}

$$
\begin{aligned}
&{ }^{2} \rho_{a i}={ }^{2} \rho_{a i}^{G}+{ }^{2} \rho_{a i}^{H}+{ }^{2} \rho_{a i}^{I}, \\
&{ }^{1} \rho_{a i b j}=\left({ }^{1} \rho_{a i b j}^{A}+{ }^{1} \rho_{a i b j}^{B}+P^{a b j}\left\{1 \rho_{a i b j}^{C}+{ }^{1} \rho_{a i b j}^{D}+{ }^{1} \rho_{a i b j}^{E}\right\}\right. \\
&\left.+{ }^{1} \rho_{a i b j}^{F}\right)\left(1+\delta_{a b} \delta_{i j}\right) \\
&-1 \\
&{ }^{2} \rho_{a i b j}=\left({ }^{2} \rho_{a i b j}^{A}+{ }^{2} \rho_{a i b j}^{B}+P^{a b j}\left\{{ }^{2} \rho_{a i b j}^{C}+{ }^{2} \rho_{a i b j}^{D}+{ }^{2} \rho_{a i b j}^{E}\right\}\right) \\
& \times\left(1+\delta_{a b} \delta_{i j}\right)^{-1} .
\end{aligned}
$$

The explicit forms of the various contributions are given in Table I in terms of $R_{2}$ and $T_{2}$ amplitudes and integrals of the $T_{1}$ and $R_{1}$ dependent effective Hamiltonians. Note that in deriving and implementing these equations, it is convenient to expand the $R_{2}$ and $T_{2}$ amplitudes to a squared ( $a i b j$ ) rather than packed $(a i \geqslant b j)$ form

$$
R_{2}=\sum_{a i \geqslant b j} R_{i j}^{a b} E_{a i} E_{b j}=\frac{1}{2} \sum_{a i b j}\left(1+\delta_{a b} \delta_{i j}\right) R_{i j}^{a b} E_{a i} E_{b j}
$$

and similarly for $T_{2}$. The diagonal factors have been absorbed into the amplitudes in the equations in Table I by means of the substitutions

$$
\begin{aligned}
& \left(1+\delta_{a b} \delta_{i j}\right) R_{i j}^{a b} \rightarrow R_{i j}^{a b}, \\
& \left(1+\delta_{a b} \delta_{i j}\right) t_{i j}^{a b} \rightarrow t_{i j}^{a b} .
\end{aligned}
$$

We obtain two sets of equations of a structure closely related to that of the CCSD amplitude equations. We would therefore expect the computational cost of one linear transformation in an MO-driven algorithm to be about twice that of one iteration in the CCSD amplitude equations. We shall now consider the implementation in more detail with the aim to reduce the computational cost further and also to develop a procedure compatible with the recently developed integraldriven CC code.

\section{Atomic-orbital integral-driven linear transformation}

Let us briefly review the integral-direct CCSD energy algorithm. The AO integrals are calculated in distributions with three free $\mathrm{AO}$ indices and one fixed $\mathrm{AO}$ index $\delta$ :

$$
I_{\alpha \beta, \gamma}^{\delta}=(\alpha \beta \mid \gamma \delta), \quad \alpha \geqslant \beta .
$$

All distributions belonging to the same shell are calculated simultaneously and then written to disk. The distributions are subsequently read back in one at a time in a loop over the $\delta$ index belonging to the shell in question. Inside the $\delta$ loop, one integral distribution is kept in core together with a packed result vector and the $T_{2}$ amplitudes in the squared form and also some minor intermediates. The total memory requirement in the integral-direct calculation of the CCSD amplitude equations is of the order $1 / 2 N^{3}+3 / 2 V^{2} O^{2}$. The 
TABLE II. Contributions to the linear transformation vector in terms of intermediates, reference amplitudes and trial vector amplitudes.

$$
\begin{array}{lc}
\tilde{\rho}_{a i}^{E 1}=\sum_{c} R_{i}^{c} E_{a c}^{1} & \rho_{a i}^{H}=-\sum_{d k l} R_{k l}^{a d} \hat{L}_{l d k i} \\
\tilde{\rho}_{a i}^{E 2}=-\sum_{k} R_{k}^{a} E_{k i}^{2} & \rho_{a i}^{G}=\sum_{c d k} R_{i k}^{c d} \hat{L}_{k d a c} \\
\tilde{\rho}_{a i}^{I}=-\sum_{c k}\left(2 t_{i k}^{a c}-t_{i k}^{c a}\right) \widetilde{F}_{k c} & \rho_{a i}^{I}=\sum_{c k}\left(2 R_{i k}^{a c}-R_{i k}^{c a}\right) \hat{F}_{k c} \\
\tilde{\rho}_{a i}^{J}=\widetilde{F}_{a i}^{*} & \rho_{a i b j}^{A}=\sum_{k l} R_{k l}^{a b} \Gamma_{k i l j} \\
\tilde{\rho}_{a i b j}^{A}=\sum_{k l} t_{k l}^{a b} \widetilde{\Gamma}_{k i l j} & \rho_{a i b j}^{B F}=\sum_{\alpha \beta}\left(\bar{\Lambda}_{\alpha a}^{p} \Lambda_{\beta b}^{p}+\Lambda_{\alpha a}^{p} \bar{\Lambda}_{\beta b}^{p}\right) \rho_{\alpha i \beta j}^{B F} \\
\tilde{\rho}_{a i b j}^{B F}=\sum_{\alpha \beta} \Lambda_{\alpha a}^{p} \Lambda_{\beta b}^{p} \widetilde{\rho}_{\alpha i \beta j}^{B F} & \rho_{a i b j}^{C}=-\left(\frac{1}{2}+P_{i j}\right) \sum_{c k} R_{j k}^{c b} C_{c k a i} \\
\tilde{\rho}_{a i b j}^{C}=-\left(\frac{1}{2}+P_{i j}\right) \sum_{k c} t_{j k}^{c b} \widetilde{C}_{c k a i} & \rho_{a i b j}^{D}=\frac{1}{2} \sum_{c k}\left(2 R_{j k}^{b c}-R_{j k}^{c b}\right) D_{c k a i} \\
\tilde{\rho}_{a i b j}^{D}=\frac{1}{2} \sum_{c k}\left(2 t_{j k}^{b c}-t_{j k}^{c b}\right) \widetilde{D}_{c k a i} & \rho_{a i b j}^{E 1}=\sum_{c} R_{i j}^{a c} E_{b c}^{1} \\
\tilde{\rho}_{a i b j}^{E 1}=\sum_{c} t_{i j}^{a c} \widetilde{E}_{b c}^{1} & \rho_{a i b j}^{E 2}=-\sum_{k} R_{i k}^{a b} E_{k j}^{2} \\
\tilde{\rho}_{a i b j}^{E 2}=-\sum_{k} t_{i k}^{a b} \widetilde{E}_{k j}^{2} &
\end{array}
$$

scratch space required is of the order of a few times (2-30) this number. This scratch space is used for storage of integral distributions, some intermediates, and the CCSD trial vectors in the iterative algorithm.

In designing an efficient algorithm for the calculation of the linearly transformed vectors in CCSD, it is important to consider the operation count and the vectorization of the code as well as the memory and scratch-space requirements. Significant reductions in the operation count can be achieved by the use of global and local intermediates. Global intermediates do not depend on the trial vector coefficients-they are constructed once and for all, written to disk and then read back in when needed. Local intermediates on the other hand depend on $R_{1}$ and $R_{2}$-they are calculated in each linear

\begin{tabular}{|c|c|}
\hline Global Intermediates & Local Intermediates \\
\hline$\rho_{\alpha i \beta j}^{B F}=(\alpha i \mid \beta j)+\sum_{c d} t_{i j}^{c d}(\alpha c \hat{\mid \beta d})$ & $\widetilde{\rho}_{\alpha i \beta j}^{B F}=(\alpha i \tilde{\mid} \beta j)+\sum_{c d} R_{i j}^{c d}(\alpha c \hat{\mid} \beta d)$ \\
\hline$\Gamma_{k i l j}=(k i \mid \hat{l} l j)+\sum_{c d} t_{i j}^{c d}(k c \mid \hat{l} d)$ & $\widetilde{\Gamma}_{k i l j}=(k i \widetilde{\mid l j})+\sum_{c d} R_{i j}^{c d}(k c \mid l d)$ \\
\hline$C_{c k a i}=(k i \hat{\mid} \mid a c)-\sum_{d l} t_{l i}^{a d}(l c \mid k d)$ & $\widetilde{C}_{c k a i}=(k i \widetilde{\mid a c})=(k i \hat{\mid} \mid a c)+(k i \hat{\mid} \overline{a c})$ \\
\hline$D_{c k a i}=\hat{L}_{k c a i}+\sum_{d l}\left(2 t_{i l}^{a d}-t_{i l}^{d a}\right) L_{l d k e}$ & $\widetilde{D}_{c k a i}=\widetilde{L}_{k c a i}=\hat{L}_{k c a \bar{a}}+\hat{L}_{k c a} \bar{i}$ \\
\hline$E_{b c}^{1}=\hat{F}_{b c}-\sum_{d l m} t_{l m}^{d b} L_{l d m c}$ & $\widetilde{E}_{b c}^{1}=\widetilde{F}_{b c}-\sum_{d l m} R_{l m}^{d b} L_{l d m e}$ \\
\hline$E_{k j}^{2}=\hat{F}_{k j}+\sum_{d e m} t_{j m}^{d e} L_{m e k d}$ & $\widetilde{E}_{k i}^{2}=\widetilde{F}_{k i}+\sum_{d e m} R_{i m}^{d e} L_{m e k d}$ \\
\hline$\hat{F}_{p q}=\hat{h}_{p q}+\sum_{k} \hat{L}_{p q k k}$ & $\begin{array}{c}\widetilde{F}_{p q}^{*}=\sum_{k} \hat{L}_{p q k \bar{k}} \\
\widetilde{F}_{p q}=\widetilde{F}_{p q}^{*}+\hat{F}_{\bar{p} q}+\hat{F}_{p \bar{q}}\end{array}$ \\
\hline
\end{tabular}
transformation and either written to disk or kept in memory. The use of intermediates is to some degree a compromise between memory and scratch space requirements and computational cost.

To avoid introducing new limitations on the size of the systems that can be handled (above those already inherent in the integral-direct CCSD model for the calculation of the ground state energy), we now require that the additional scratch space requirements from the use of intermediates should not exceed a few times $V^{2} O^{2}$. In most applications with reasonably accurate basis sets, $O$ is considerably smaller than $N$. Similarly, the memory requirements for the calculation of the linearly transformed vector should be comparable to those of the CCSD energy code. Concerning the vectorization, we rely on our previous experience with the implementation of the CCSD amplitude equations. ${ }^{26,27} \mathrm{We}$ now manipulate the formulas in Table I to obtain the linearly
TABLE III. Intermediates in CCSD linear transformation from the right.

transformed vector in accordance with the above discussion. The results are given in Tables II and III. We discuss the most important manipulations below.

Since we carry out transformations with $R_{1}$ and $R_{2}$ simultaneously, we may combine the ${ }^{1} \rho_{a i b j}^{F},{ }^{1} \rho_{a i b j}^{B}$ and ${ }^{2} \rho_{a i b j}^{B}$ terms of the transformed vector

$$
\begin{aligned}
{ }^{2} \rho_{a i b j}^{B}+{ }^{1} \rho_{a i b j}^{B}+{ }^{1} \rho_{a i b j}^{F} \\
=\sum_{\alpha \beta}\left(\Lambda_{\alpha a}^{p} \Lambda_{\beta b}^{p}\right)\left(\sum_{c d} R_{i j}^{c d}(\alpha c \hat{\mid} \beta d)\right) \\
+\sum_{\alpha \beta}\left(\bar{\Lambda}_{\alpha a}^{p} \Lambda_{\beta b}^{p}+\Lambda_{\alpha a}^{p} \bar{\Lambda}_{\beta b}^{p}\right) \\
+\left(\sum_{c d} t_{i j}^{c d}(\alpha c \hat{\mid} \beta d)\right) \\
+\sum_{\alpha \beta}\left(\Lambda_{\alpha a}^{p} \Lambda_{\beta b}^{p}\right)((\alpha \bar{i} \hat{\mid} \beta j)+(\alpha i \hat{\imath} \beta \bar{j})) \\
+\sum_{\alpha \beta}\left(\bar{\Lambda}_{\alpha a}^{p} \Lambda_{\beta b}^{p}+\Lambda_{\alpha a}^{p} \bar{\Lambda}_{\beta b}^{p}\right)(\alpha i \hat{\mid} \beta j) .
\end{aligned}
$$

We may rewrite this expression in terms of the two contributions

$$
\begin{aligned}
\rho_{a i b j}^{B F}= & \sum_{\alpha \beta}\left(\bar{\Lambda}_{\alpha a}^{p} \Lambda_{\beta b}^{p}+\Lambda_{\alpha a}^{p} \bar{\Lambda}_{\beta b}^{p}\right)((\alpha i \hat{\mid} \beta j) \\
& \left.+\sum_{c d} t_{i j}^{c d}(\alpha c \hat{\mid} \beta d)\right),
\end{aligned}
$$


TABLE IV. Operation count of terms in CCSD linear transformation. Nomenclature refers to Tables II and III. Only the most dominant operation count in each term is reported.

\begin{tabular}{lccc}
\hline \hline & $\begin{array}{c}\text { MO-algorithm } \\
\text { without intermediates }\end{array}$ & $\begin{array}{c}\text { MO-algorithm } \\
\text { with intermediates }\end{array}$ & $\begin{array}{c}\text { AO-algorithm } \\
\text { with intermediates }\end{array}$ \\
\hline$\Gamma$-intermediate & $1 / 2 \mathrm{~V}^{2} \mathrm{O}^{4}$ & $1 / 4 \mathrm{~V}^{2} \mathrm{O}^{4}$ & $\mathrm{NO}(\mathrm{N}+\mathrm{O})$ \\
$A$-terms & $\mathrm{V}^{2} \mathrm{O}^{4}$ & $\mathrm{~V}^{2} \mathrm{O}^{4}$ & $\mathrm{~V}^{2} \mathrm{O}^{4}$ \\
$B F$-terms & $1 / 4 \mathrm{~V}^{4} \mathrm{O}^{2}+\mathrm{V}^{3} \mathrm{O}^{3}$ & $1 / 4 \mathrm{~V}^{4} \mathrm{O}^{2}$ & $1 / 4 \mathrm{~N}^{4} \mathrm{O}^{2}+4 \mathrm{~N}^{3} \mathrm{O}^{2}$ \\
$C$-terms & $3 \mathrm{~V}^{3} \mathrm{O}^{3}$ & $2 \mathrm{~V}^{3} \mathrm{O}^{3}$ & $2 \mathrm{~V}^{3} \mathrm{O}^{3}$ \\
$D$-terms & $3 \mathrm{~V}^{3} \mathrm{O}^{3}$ & $2 \mathrm{~V}^{3} \mathrm{O}^{3}$ & $2 \mathrm{~V}^{3} \mathrm{O}^{3}$ \\
$E$-terms & $4 \mathrm{~V}^{3} \mathrm{O}^{2}$ & $2 \mathrm{~V}^{3} \mathrm{O}^{2}$ & $\mathrm{~N}^{2} \mathrm{VO}^{2}$ \\
$G$-terms & $\mathrm{V}^{3} \mathrm{O}^{2}$ & $\mathrm{~V}^{3} \mathrm{O}^{2}$ & $\mathrm{~N}^{2} \mathrm{O}^{2}+\mathrm{N}^{2} \mathrm{VO}$ \\
$H$-terms & $\mathrm{V}^{2} \mathrm{O}^{3}$ & $\mathrm{~V}^{2} \mathrm{O}^{3}$ & $\mathrm{NVO}^{3}$ \\
$I$-terms & $\mathrm{V}^{2} \mathrm{O}^{2}$ & $\mathrm{~V}^{2} \mathrm{O}^{2}$ & $\mathrm{~V}^{2} \mathrm{O}^{2}$ \\
$J$-terms & $\mathrm{VO}^{3}$ & $\mathrm{VO}^{3}$ & $\mathrm{VO}^{3}$ \\
Sum & $1 / 4 \mathrm{~V}^{4} \mathrm{O}^{2}+7 \mathrm{~V}^{3} \mathrm{O}^{3}+3 / 2 \mathrm{~V}^{2} \mathrm{O}^{4}$ & $1 / 4 \mathrm{~V}^{4} \mathrm{O}^{2}+4 \mathrm{~V}^{3} \mathrm{O}^{3}+5 / 4 \mathrm{~V}^{2} \mathrm{O}^{4}$ & $1 / 4 \mathrm{~N}^{4} \mathrm{O}^{2}+4 \mathrm{~V}^{3} \mathrm{O}^{3}+\mathrm{V}^{2} \mathrm{O}^{4}$ \\
\hline \hline
\end{tabular}

${ }^{a}$ Intermediates are restricted to having a maximum size of order $\mathrm{N}^{2} \mathrm{O}^{2}$.

$$
\begin{aligned}
\tilde{\rho}_{a i b j}^{B F}= & \sum_{\alpha \beta}\left(\Lambda_{\alpha a}^{p} \Lambda_{\beta b}^{p}\right)((\alpha \bar{i} \hat{\mid} \beta j)+(\alpha i \hat{\mid} \beta \bar{j}) \\
& \left.+\sum_{c d} R_{i j}^{c d}(\alpha c \hat{\mid} \beta d)\right)
\end{aligned}
$$

In this way, we have rewritten the three contributions in two terms. We identify the term in the second set of parentheses in Eq. (64) as a global intermediate, equivalent to the corresponding term in the AO-driven calculation of the $B$ and $F$ intermediates in Ref. 27.

$$
\Omega_{\alpha i \beta j}^{B F}=(\alpha i \hat{\mid} \beta j)+\sum_{c d} t_{i j}^{c d}(\alpha c \hat{\mid} \beta d) .
$$

In Ref. 27, it is demonstrated how this term can be implemented in a fully AO-driven approach and with a minimal operation count of $1 / 4 N^{4} O^{2}$. Having constructed this intermediate once, we need only perform the additional contraction in Eq. (64) in $N^{2} O^{2}(N+V)$ operations. The other $B / F$ term in Eq. (65) is constructed using a local intermediate similar to Eq. (66) and by means of a similar contraction. We have thus obtained savings in the operation count of about $V^{3} O^{3}$.

Consider the $A$ terms

$$
\begin{aligned}
{ }^{2} \rho_{a i b j}^{A}+{ }^{1} \rho_{a i b j}^{A}= & \sum_{k l} t_{k l}^{a b}((k \bar{i} \hat{\mid} 1 j)+(k i \hat{\mid} 1 \bar{j}) \\
& \left.+\sum_{c d} R_{i j}^{c d}(k c \hat{\mid} 1 d)\right) \\
& +\sum_{k l} R_{k l}^{a b}\left((k i \hat{\mid} \mid j)+\sum_{c d} t_{i j}^{c d}(k c \hat{\mid} 1 d)\right) .
\end{aligned}
$$

The term inside the second pair of parentheses is identical to the $\Gamma$ intermediate in the energy code (see Table II) whereas the term inside the first pair of parentheses corresponds to the generalized trial-vector dependent local $\Gamma$ intermediate. We may thus write the $A$ term as a contribution from a global intermediate and as a contribution from a local intermediate. In Ref. 27 it was demonstrated how the global $\Gamma$ intermediate can be obtained from $\rho_{\alpha i \beta j}^{B F}$. We find here in a similar way that the local intermediate can be obtained from $\tilde{\rho}_{\alpha i \beta j}^{B F}$ since

$$
\begin{aligned}
\widetilde{\Gamma}_{k i l j}= & \sum_{\alpha \beta}\left(\Lambda_{\alpha k}^{p} \Lambda_{\beta l}^{p}\right)((\alpha \bar{i} \hat{\mid} \beta j)+(\alpha i \hat{\mid} \beta \bar{j}) \\
& \left.+\sum_{c d} R_{i j}^{c d}(\alpha c \hat{\mid} \beta d)\right) .
\end{aligned}
$$

We may carry on with similar manipulations. In Table II we give the expression of the various contributions in terms of the global and local intermediates in Table III. Local intermediates depending on $R$ and the terms involving these intermediates are distinguished from the remaining terms by a tilde. We have discussed a few of these terms only and for the sake of conciseness give only a few general remarks on the evaluation of the remaining terms.

(1) The global intermediates are identical to the intermediates for the CCSD amplitude equations with the trivial exception of a factor of 1 in front of the second term in the $C$ and $D$ intermediates rather than a factor of $\frac{1}{2}$ as in the energy code. On the other hand, the local intermediates $\widetilde{C}$ and $\widetilde{D}$ have no $t_{2}$ contributions. This way of writing the terms is possible for the $C$ and $D$ terms since these terms are symmetric to interchange of $R_{2}$ and $T_{2}$ amplitudes and it is convenient since it saves contractions of $t_{2}$ with integrals $\left(V^{3} O^{3}\right)$. This is not the case for the other bilinear contributions to the $E$ and the $A$ terms and instead of storing very large intermediates of the size $V^{3} O$ and $V^{4}$, we calculate the $E$ and $A$ terms by one local and one global intermediate. We demonstrated above how the local $\Gamma$ intermediate can be constructed easily from the local $B / F$ intermediate. The calculation of the $E$ intermediate scales as $N^{2} V^{2} O$. The calculation of the $E$ and $A$ intermediates is thus far from being the most time consuming part of a CCSD linear transformation.

(2) The local intermediates are analogous with the global intermediates with the extra complication that the integrals may be non-totally symmetric and may contain several contributions. Consider for example the $\widetilde{C}$ intermediate. The two terms are constructed in a loop over $\delta$ as 


$$
\widetilde{C}_{\delta k a i}=(k i \hat{\mid} a \delta)=(k \bar{i} \hat{\mid} a \delta)+(k i \hat{\imath} \bar{a} \delta)
$$

For a fixed index $\delta$, the two terms are calculated and then added together and subsequently written to disk as one intermediate. As for the $C$ intermediate, the $\widetilde{C}$ intermediate is read in, the $\delta$ index transformed to MO basis, and the resulting MO intermediate contracted with $T_{2}$ and added to the result vector. The last part is done in a loop over batches of indices $(a i)$ in order to obtain the highest possible vector lengths without increasing the memory requirements. A similar discussion applies to the $D$ terms.

(3) The terms arising in the transformation by the singles-singles block of the Jacobian have been expressed in terms of $E$ intermediates. In this way we avoid having both $T_{2}$ and $R_{2}$ in memory inside the $\delta$ loop, which would be necessary if these contributions were calculated as $G$ and $H$ terms in Table I. Additional operation counts of the order $N^{3} \mathrm{O}^{2}$ are thus avoided, which is particularly important for the $\mathrm{CC} 2$ model.

(4) Using the expressions in Tables II and III, we obtain the bilinear contributions as terms with contraction of $T_{2}$ with local intermediates and $R_{2}$ with global intermediates. Accepting IO of $T_{2}$ and $R_{2}$ amplitudes after the $\delta$ loop, we may divide the contractions into a section with $T_{2}$ in memory and a section with $R_{2}$ in memory.

The algorithm for calculating integral-direct excitation energies can be summarized as follows:

(1) Calculate reference amplitudes in the integral-direct technique of Refs. 26, 27.

(2) Calculate global intermediates:

$$
\rho_{\alpha i \beta j}^{B F}, C_{c k a i}, D_{c k a i}, E_{k i}^{2}, E_{b c}^{1}, \hat{F}_{p q} .
$$

(3) Excitation energy calculation-a generalized Davidson algorithm $^{74}$ combined with the calculation of linearly transformed vectors in an integral-direct algorithm:

(A) Construction of auxiliary matrices:

$$
\Lambda_{\alpha p}^{h}, \Lambda_{\alpha p}^{p}, \bar{\Lambda}_{\alpha p}^{h}, \bar{\Lambda}_{\alpha p}^{p}, \text { etc. }
$$

(B) Loop over first atomic index $\delta$. Add contributions to intermediates and result-vectors:

$\rho_{a i}^{G}, \rho_{a i}^{H}$,

$\tilde{\rho}_{\alpha i \beta j}^{B F}, \widetilde{C}_{c k a i}, \widetilde{D}_{c k a i}, \widetilde{E}_{k i}^{2}, \widetilde{E}_{b \delta}^{1}, \widetilde{F}_{\alpha \beta}^{*}$,

In memory: ${ }^{1} \widetilde{\rho}_{\alpha i \beta j}^{B F}, R_{k l}^{c d}$ and $(\alpha \beta \gamma \delta) ; 3 / 2 V^{2} O^{2}$

$+1 / 2 N^{3}$.

(C) Transform to MO-basis:

$\tilde{\rho}_{a i b j}^{B F}, \rho_{a i b j}^{B F}, \widetilde{F}_{p q}^{*}, \widetilde{F}_{p q}$.

(D) Read in global intermediates and contract with $\left(R_{k}^{c}, R_{k l}^{c d}\right)$ :

$\rho_{a i}^{I}, \quad \tilde{\rho}_{a i}^{E 1}, \tilde{\rho}_{a i}^{E 2}, \tilde{\rho}_{a i}^{J}$, $\rho_{a i b j}^{C}, \rho_{a i b j}^{D}, \rho_{a i b j}^{E 1}, \rho_{a i b j}^{E 2}, \rho_{a i b j}^{A 2}$,

In memory: $\rho_{a i b j}, R_{k l}^{c d} ; 3 / 2 V^{2} O^{2}$.

(E) Contract local intermediates with $t_{k l}^{c d}$ :

$$
\begin{aligned}
& \tilde{\rho}_{a i}^{I}, \\
& \tilde{\rho}_{a i b j}^{C}, \quad \tilde{\rho}_{a i b j}^{D}, \tilde{\rho}_{E 1}^{a i b j}, \tilde{\rho}_{a i b j}^{E 2}, \quad \widetilde{\rho}_{a i b j}^{A},
\end{aligned}
$$

In memory: $\rho_{a i b j}, t_{k l}^{c d} ; 3 / 2 V^{2} O^{2}$.

In the specifications of the memory requirements, we have only included the most important vectors with requirements larger than $\mathrm{N}^{2} \mathrm{O}$. In this algorithm, the maximum memory requirements are inside the loop over one atomic index, where we need to keep the ${ }^{1} \tilde{\rho}_{\alpha i \beta j}^{B F}$ and $R_{k l}^{c d}$ together with the integral distribution. After this loop, we may perform IO on $t_{2}$ and $R_{2}$ amplitudes. The algorithm requires that the amplitude vectors are stored as the full $(a i, b j)$ matrix while the result vector may be packed as $(a i \geqslant b j)$. We thus have a memory requirement of the order of $3 / 2 V^{2} O^{2}+1 / 2 N^{3}$, which is similar to the one for optimizing the $\mathrm{CC}$ state.

In addition, there are options for saving CPU time when scratch, memory and IO conditions allow this to happen. The $R_{2}$ amplitudes with the occupied indices transposed may be kept in memory saving, CPU time in calculation of the $E$ intermediates. There is also the option for performing more linear transformations in one integral calculation. The integral calculation is performed in a loop over shells and for each shell we may perform I/O on trial vectors:

Loop shells

Calculate integrals

Loop Trial vectors

Loop AO-index

Calculate

End Loop

\section{End Loop}

\section{End Loop}

This scheme save recalculations of integrals at the cost of increased I/O. In integral-direct calculations, the recalculation of integrals may take a significant part of the total CPU time, in particular for the CC2 model.

In order to compare the efficiency of the AO and the MO integral based algorithms we have in Table IV give the operator count for the individual terms in the two algorithms.

\section{E. The CC2 model}

We introduce a partitioning of the Hamiltonian $H$ into a Fock operator $F$ and a fluctuation operator $U$ that describes the difference between the true electron-electron repulsion and the Fock potential:

$$
H=F+U \text {. }
$$

CC2 is defined as an approximation to CCSD as follows: The CCSD singles equations Eq. (28) are retained in their original form but the doubles equations Eq. (29) are approxi- 
mated to be correct through first order only with the singles treated as zero-order parameters. ${ }^{15}$ The $\mathrm{CC} 2$ equations are thus given by

$$
\begin{aligned}
& \left\langle\mu_{1}\left|\hat{H}+\left[\hat{H}, T_{2}\right]\right| H F\right\rangle=0, \\
& \left\langle\mu_{2}\left|\left[F, T_{2}\right]+\hat{H}\right| H F\right\rangle=0 .
\end{aligned}
$$

The CC2 doubles equations given an MP2 like expression, but with $T_{1}$ transformed integrals:

$$
\left(\epsilon_{a}+\epsilon_{b}-\epsilon_{i}-\epsilon_{j}\right) t_{i j}^{a b}+(a i \hat{\mid} b j)=0 .
$$

The CC2 response function is given in Ref. 15. Excitation energies and transition moments are determined from the poles and residues of the linear response function. The $\mathrm{CC} 2$ excitation energies are in accordance with Eqs. (10) and (12) determined from the eigenvalue equation Eq. (10) where the CC2 Jacobian is

$$
A_{\mu_{i} \nu_{j}}=\left(\begin{array}{cc}
\left\langle\mu_{1}\left|\left[\hat{H}, t_{\nu_{1}}\right]+\left[\left[\hat{H}, \tau_{\nu_{1}}\right], T_{2}\right]\right| H F\right\rangle & \left\langle\mu_{1}\left|\left[\hat{H}, \tau_{\nu_{2}}\right]\right| H F\right\rangle \\
\left\langle\mu_{2}\left|\left[\hat{H}, \tau_{\nu_{1}}\right]\right| H F\right\rangle & \delta_{\mu \nu} \omega_{\mu_{2}}
\end{array}\right) .
$$

The doubles-doubles block is a simple diagonal consisting of orbital energy differences. The CC2 linear transformed vector thus becomes

$$
\begin{aligned}
& { }^{1} \rho_{a i}=\left\langle\begin{array}{c}
a \\
i
\end{array}|\widetilde{H}| H F\right\rangle+\left\langle\begin{array}{c}
a \\
i
\end{array}\left|\left[\widetilde{H}, T_{2}\right]\right| H F\right\rangle, \\
& { }^{1} \rho_{a i b j}=\left\langle\begin{array}{c}
a b \\
i j
\end{array}|\widetilde{H}| H F\right\rangle, \\
& { }^{2} \rho_{a i}=\left\langle\begin{array}{c}
a \\
i
\end{array}\left|\left[\hat{H}, R_{2}\right]\right| H F\right\rangle, \\
& { }^{2} \rho_{a i b j}=\left\langle\begin{array}{c}
a b \\
i j
\end{array}\left|\left[F, R_{2}\right]\right| H F\right\rangle .
\end{aligned}
$$

${ }^{1} \rho_{a i}$ and ${ }^{2} \rho_{a i}$ are calculated as for the CCSD model, ${ }^{2} \rho_{a i b j}$ is a trivial multiplication of the doubles amplitudes with orbital energies. The calculation of the ${ }^{1} \rho_{a i b j}$ vectors is performed with direct transformation to $\mathrm{MO}$ basis as

$$
\begin{aligned}
{ }^{1} \rho_{a i b j}= & (a i \hat{\mid} b j)=(\overline{a i} \hat{\mid} \mid b j)+(a \bar{i} \hat{\mid} b j)+(a i \hat{\mid} \bar{b} j) \\
& +(a i \hat{\mid} b \bar{j}) .
\end{aligned}
$$

\section{BENZENE SINGLET EXCITED STATES}

\section{A. Calculations}

By calculating the singlet excitation energies of the benzene molecule, we will demonstrate that the combination of integral-direct $\mathrm{CC}$ techniques with a hierarchy of CC models opens up new possibilities for the reliable theoretical assignment of electronic excitation spectra. All calculations were carried out at the same geometry as in recent CASPT2 (Ref. 56) and SOPPA (Ref. 57) studies, which is close to the experimental geometries. Only vertical excitation energies are considered in this study. First, we discuss the convergence of the calculations with respect to the basis set. Next, the effects of correlation are studied by performing calculations in the hierarchy of coupled cluster models CCS, CC2, CCSD, and $\mathrm{CC} 3$ and also by using perturbative triples corrections
$\operatorname{CCSDR}(3)$ and $\operatorname{CCSDR}(1 a)$. The errors in the solution of the electronic problem are estimated and comparisons are made with other recent calculations. Finally, we compare our theoretical results with experiments.

All calculations were carried out on a SGI Power Challenge computer. A single linear transformation with one trial vector was observed to take slightly less time than one coupled cluster energy iteration, in agreement with the analysis of Section II. The option of performing transformations on several vectors of same symmetry in one integral calculation reduced the average CPU-time per transformed vector somewhat. The relative costs of the individual terms in the transformations are similar to those for the CCSD groundstate optimizations in Ref. 27, to which we refer for details on timings.

\section{B. Basis set investigations}

The singlet excitation energies obtained in the $\mathrm{CC} 2$ and CCSD calculations are given in Table $\mathrm{V}$ for the different basis sets. The excited states are classified as valence and as Rydberg $\pi \pi^{*}$ and $\pi \sigma^{*}$ excitations. In addition to calculations on the $n=3$ Rydberg series converging towards the first ionization potential, we also give a few results for the $n=4$ Rydberg states and for the first Rydberg state of $E_{2 g}$ symmetry, corresponding to the second ionization potential. All electrons were correlated in these calculations.

The basis-set study concentrates on extensions of the aug-cc- $p$ VDZ basis set. ${ }^{75,76}$ Additional diffuse functions were placed at the center of mass (CM) in order to describe the Rydberg states. In the previously published $\mathrm{CC} 2$ basis-set study, ${ }^{51}$ we investigated the saturation in the $\mathrm{CM}$ functions at the aug-cc- $p$ VDZ level. First, we added a set of contracted optimized atomic natural orbital (ANO) functions of Lorentzon et al. (CM8), ${ }^{56}$ where the associated uncontracted set corresponds to the universal primitive functions of Kaufmann et al. ${ }^{77}$ Next, calculations were carried out using smaller CM sets of primitive $s, p$, and $d$ functions. For the $n=3$ Rydberg series, we found that the CM2 basis-which contains two sets of primitive $s, p$, and $d$ functions with 
TABLE V. $\mathrm{C}_{6} \mathrm{H}_{6}$ singlet excitation energies in $\mathrm{eV}$ and the percentage of single excitation in the right excitation vectors obtained in CCSD. ${ }^{\mathrm{a}}$

\begin{tabular}{|c|c|c|c|c|c|c|c|}
\hline \multirow{3}{*}{$\begin{array}{l}\text { One electron Basis: } \\
\text { CC-Model }\end{array}$} & \multicolumn{2}{|c|}{$\mathrm{ANO}^{\mathrm{b}}$} & \multicolumn{2}{|c|}{ aug-cc-pVDZ-CM2 ${ }^{c}$} & \multicolumn{3}{|c|}{ aug-cc-pVTZ-CM2 ${ }^{\mathrm{d}}$} \\
\hline & \multirow{2}{*}{$\begin{array}{l}\mathrm{CC} 2 \\
\Delta E\end{array}$} & \multirow{2}{*}{$\begin{array}{l}\text { CCSD } \\
\Delta E\end{array}$} & \multirow{2}{*}{$\begin{array}{l}\mathrm{CC} 2 \\
\Delta E\end{array}$} & \multirow{2}{*}{$\begin{array}{c}\text { CCSD } \\
\Delta E\end{array}$} & \multirow{2}{*}{$\begin{array}{l}\mathrm{CC} 2 \\
\Delta E\end{array}$} & \multicolumn{2}{|c|}{ CCSD } \\
\hline & & & & & & $\Delta E$ & $\% \mathrm{~T}$ \\
\hline $1^{1} B_{2 u}\left(V-\pi \pi^{*}\right)$ & 5.265 & 5.189 & 5.274 & 5.192 & 5.232 & 5.180 & 91 \\
\hline $1^{1} B_{1 u}$ & 6.556 & 6.590 & 6.502 & 6.537 & 6.463 & 6.481 & 95 \\
\hline $1^{1} E_{1 u}$ & 7.018 & 7.171 & 6.991 & 7.148 & 7.070 & 7.227 & 95 \\
\hline $2{ }^{1} E_{2 g}$ & 8.967 & 9.177 & 8.951 & 9.157 & 8.909 & 9.168 & 87 \\
\hline $2{ }^{1} E_{1 u}\left(R-\pi \pi^{*}, n=3\right)$ & 7.371 & 7.579 & 7.283 & 7.481 & 7.319 & 7.501 & 95 \\
\hline $2{ }^{1} A_{1 g}$ & 7.656 & 7.855 & 7.644 & 7.856 & 7.806 & 7.984 & 96 \\
\hline $1{ }^{1} E_{2 g}$ & 7.651 & 7.847 & 7.638 & 7.844 & 7.798 & 7.972 & 95 \\
\hline $1{ }^{1} A_{2 g}$ & 7.681 & 7.880 & 7.667 & 7.879 & 7.829 & 8.009 & 95 \\
\hline $1{ }^{1} E_{1 g}\left(R-\pi \sigma^{*}, n=3\right)$ & 6.403 & 6.547 & 6.304 & 6.440 & 6.452 & 6.563 & 95 \\
\hline $1{ }^{1} A_{2 u}$ & 6.838 & 6.988 & 6.811 & 6.964 & 6.970 & 7.092 & 95 \\
\hline $1{ }^{1} E_{2 u}$ & 6.892 & 7.056 & 6.868 & 7.036 & 7.028 & 7.169 & 95 \\
\hline $1^{1} A_{1 u}$ & 6.962 & 7.139 & 6.943 & 7.126 & 7.120 & 7.262 & 96 \\
\hline $1{ }^{1} B_{2 g}$ & 7.466 & 7.659 & 7.436 & 7.626 & 7.600 & 7.760 & 95 \\
\hline $1{ }^{1} B_{1 g}$ & 7.461 & 7.660 & 7.425 & 7.617 & 7.587 & 7.751 & 95 \\
\hline $2{ }^{1} E_{1 g}$ & 7.457 & 7.639 & 7.422 & 7.588 & 7.557 & 7.671 & 95 \\
\hline $3{ }^{1} E_{1 g}$ & 7.519 & 7.698 & 7.482 & 7.656 & 7.613 & 7.772 & 95 \\
\hline $3{ }^{1} E_{2 g}$ & 8.918 & 9.413 & 8.781 & 9.251 & 8.879 & 9.387 & 95 \\
\hline \multicolumn{8}{|l|}{$\left(R-e_{2 g}^{2 g} \sigma-a_{1 g}, n=3\right)$} \\
\hline${ }^{1} E_{2 g}\left(R-\pi \pi^{*}, n=4\right)$ & & & 8.173 & 8.456 & 8.339 & 8.591 & 96 \\
\hline${ }^{1} A_{1 g}$ & & & 8.179 & 8.464 & 8.347 & 8.599 & 96 \\
\hline${ }^{1} A_{2 g}$ & & & 8.185 & 8.469 & 8.353 & 8.605 & 96 \\
\hline
\end{tabular}

${ }^{\mathrm{a}}$ Geometry as in Ref. 56. The geometry is $R_{\mathrm{CC}}=1.3950 \AA$ and $R_{\mathrm{CH}}=1.0850 \AA$. Excitation energies are converged to an uncertainty of about $0.001 \mathrm{eV}$. The blank entries are due to excitations not obtained due to basis set inadequacy. All electrons are correlated.

${ }^{\mathrm{b}}$ ANO basis set from Ref. 56: C:[1494/431], H:[84/21], CM[888/111], giving 147 contracted basis functions. Total ground state energies in hartree are $E_{H F}=-230.771810, E_{\mathrm{MP} 2}=-230.634979, E_{\mathrm{CC} 2}=-231.666327, E_{\mathrm{CCSD}}=-231.666327$. Results taken from Ref. 51.

${ }^{c}$ Augmented correlation consistent pVDZ basis set with extra center of mass functions as described in Ref. 51: C:[1052/432], H:[52/32], CM:[222/222], giving 210 contracted basis functions. Total ground state energies in Hartree are $E_{H F}=-230.728171, E_{\mathrm{MP} 2}=-231.556430, E_{\mathrm{CC} 2}=-231.563516$, $E_{\mathrm{CCSD}}=-231.591262$. CC2 results from Ref. 51.

${ }^{\mathrm{d}}$ Augmented correlation consistent pVTZ basis set with extra center of mass functions as described in Ref. 51: C:[11632/5432], H:[632/432], CM:[222/222], giving 432 contracted basis functions. Total ground state energies in Hartree are $E_{H F}=-230.780888, E_{\mathrm{MP} 2}=-231.855173, E_{\mathrm{CC} 2}=-231.864721$, $E_{\mathrm{CCSD}}=-231.881229$. CC2 results from Ref. 51.

exponents 0.01 and 0.0033 (the same exponents for all angular momenta)—gave results within $0.05 \mathrm{eV}$ of the CM8 results. To address the problem of valence-space saturation, we have here carried out calculations using an aug-cc- $p$ VTZ basis augmented with the CM2 functions. We have not carried out the full basis-set investigation at the CCSD levelonly the extension from aug-cc- $p$ VDZ-CM2 to aug-cc$p$ VTZ-CM2 was considered. In addition, we have carried out ANO calculations with the Rydberg basis set of Lorentzon et al. here referred to as ANO1.

In Table $\mathrm{V}$, we give the $\mathrm{CC} 2$ and CCSD results for these basis sets. We observe changes in the valence excitation energies ranging from -0.06 to $0.08 \mathrm{eV}$ going from aug-cc$p$ VDZ-CM2 to aug-cc- $p$ VTZ-CM2. The Rydberg excitations shift by 0.1 to $0.2 \mathrm{eV}$. The $2{ }^{1} E_{1 u}$ state constitutes an exception, with shifts of only $0.04 \mathrm{eV}$. For a balanced description of the electronic ground state and the Rydberg states, it is essential to have a good description of the valence region and of the penetration of the Rydberg electron.

Except for $3{ }^{1} E_{2 g}$, all Rydberg states have the same cationic core. The constant difference between aug-cc- $p$ VDZ$\mathrm{CM} 2$ and aug-cc- $p$ VTZ-CM2 for these states suggests that the added flexibility of the aug-cc- $p$ VTZ-CM2 basis is important for accurate calculations of excitations to Rydberg states. We conclude that a basis set of augmented double- zeta polarization quality may give errors of the order $0.1-0.2$ $\mathrm{eV}$, even when supplemented with CM functions. This finding confirms the results of the $\mathrm{CC} 2$ basis set investigation. We note that the ANO1 basis set gives results of augmented double-zeta quality. The different behavior of the $2{ }^{1} E_{1 u}$ state in this respect is one of several indications that this state is not a "pure" Rydberg state. Since the $3{ }^{1} E_{2 g}$ state has a different cationic core, its behavior is expected to be different. Furthermore, there may be some mixing of this state with the valence $2{ }^{1} E_{2 g}$ state.

\section{Correlation effects}

In Table VI, we report the ANO1 singlet excitation energies of benzene using CCS, CC2, CCSD, and CC3. The results obtained from perturbative triples approaches CCSDR(3) are also listed. In the calculations in Table VI, we have frozen the core electrons (using canonical Hartree-Fock orbitals). Comparing the CC2 and CCSD results of Table VI with the calculations in Table $\mathrm{V}$ (where the core electrons are not frozen), we find that the effect of freezing the core electrons is less than $0.01 \mathrm{eV}$. Significant computational savings are obtained by freezing the core electrons in correlated cal- 
TABLE VI. $\mathrm{C}_{6} \mathrm{H}_{6}$ singlet excitation energies in $\mathrm{eV}$ for transitions to valence and $n=3$ Rydberg states. ${ }^{a}$ Weight of singles part of right excitation vector in CCSD.

\begin{tabular}{|c|c|c|c|c|c|c|c|}
\hline Method & $\begin{array}{l}\text { CCS } \\
\text { (f.c.) }\end{array}$ & $\begin{array}{l}\text { CCS } \\
\text { (f.c.) }\end{array}$ & $\begin{array}{c}\text { CCSD } \\
\text { (f.c.) }\end{array}$ & $\begin{array}{l}\text { CC3 } \\
\text { (f.c.) }\end{array}$ & $\begin{array}{c}\operatorname{CCSDR}(3) \\
\text { (f.c.) }\end{array}$ & $\begin{array}{c}\% \mathrm{~T}_{1} \\
\text { (CCSD f.c.) }\end{array}$ & $\begin{array}{c}\% \mathrm{~T}_{1} \\
\text { (CC3 f.c.) }\end{array}$ \\
\hline $1^{1} B_{2 u}\left(V-\pi \pi^{*}\right)$ & 6.028 & 5.268 & 5.189 & 5.078 & 5.120 & 91 & 86 \\
\hline $1^{1} B_{1 u}$ & 6.194 & 6.556 & 6.590 & 6.540 & 6.558 & 95 & 93 \\
\hline $1{ }^{1} E_{1 u}$ & 7.176 & 7.013 & 7.166 & 7.132 & 7.150 & 95 & 93 \\
\hline $2{ }^{1} E_{2 g}$ & 10.584 & 8.970 & 9.174 & 8.409 & 8.691 & 85 & 66 \\
\hline $2{ }^{1} E_{1 u}\left(R-\pi \pi^{*}\right)$ & 8.056 & 7.371 & 7.578 & 7.419 & 7.410 & 94 & 92 \\
\hline $2^{1} A_{1 g}$ & 7.778 & 7.649 & 7.849 & 7.859 & 7.863 & 95 & 93 \\
\hline $1{ }^{1} E_{2 g}$ & 7.801 & 7.644 & 7.841 & 7.850 & 7.854 & 95 & 93 \\
\hline $1{ }^{1} A_{2 g}$ & 7.856 & 7.675 & 7.874 & 7.880 & 7.884 & 95 & 93 \\
\hline $1^{1} E_{1 g}\left(R-\pi \sigma^{*}\right)$ & 6.620 & 6.397 & 6.541 & 6.513 & 6.523 & 95 & 93 \\
\hline $1^{1} A_{2 u}$ & 6.950 & 6.832 & 6.982 & 6.974 & 6.982 & 96 & 93 \\
\hline $1{ }^{1} E_{2 u}$ & 7.119 & 6.886 & 7.050 & 7.032 & 7.043 & 95 & 93 \\
\hline $1{ }^{1} A_{1 u}$ & 7.287 & 6.956 & 7.133 & 7.109 & 7.121 & 95 & 92 \\
\hline $1{ }^{1} B_{2 g}$ & 7.685 & 7.460 & 7.653 & 7.650 & 7.657 & 95 & 93 \\
\hline $1{ }^{1} B_{1 g}$ & 7.699 & 7.455 & 7.653 & 7.648 & 7.655 & 95 & 93 \\
\hline $2{ }^{1} E_{1 g}$ & 7.596 & 7.451 & 7.632 & 7.633 & 7.639 & 95 & 93 \\
\hline $3{ }^{1} E_{1 g}$ & 7.729 & 7.512 & 7.692 & 7.687 & 7.693 & 95 & 93 \\
\hline $3{ }^{1} E_{2 g}\left(R-\sigma \sigma^{*}\right)$ & 10.835 & 8.915 & 9.407 & 9.275 & 9.308 & 95 & 93 \\
\hline
\end{tabular}

${ }^{\mathrm{a}}$ Geometry as in Ref. 56: $R_{\mathrm{CC}}=1.3950 \AA$ and $R_{\mathrm{CH}}=1.0850 \AA$ A . Basis set is the ANO1 basis set see footnote to Table V. The Carbon $1 s$ core electrons are frozen in all calculations. Excitation energies are converged to about $0.001 \mathrm{eV}$. Total ground state energies in Hartree are $E_{H F}=-230.771810, E_{\mathrm{MP} 2}=-231.596300$, $E_{\mathrm{CC} 2}=-231.605665, E_{\mathrm{CCSD}}=-231.628657, E_{\mathrm{CC} 3}=-231.673378$

culations on large molecules. It should be emphasized, however, that the basis sets used here are not adequate for describing core effects.

The effect of triple excitations on the excitation energies varies significantly. For most of the Rydberg states, very small effects are observed-less than $0.02 \mathrm{eV}$. Exceptions to this rule are the $2{ }^{1} E_{1 u} \pi$ - $\pi^{*}$ Rydberg state and the $3{ }^{1} E_{2 g}$ state, where the triples contributions are as large as -0.16 $\mathrm{eV}$. For the ${ }^{1} E_{1 u},{ }^{1} B_{1 u}$, and ${ }^{1} B_{2 u}$ valence states, we also find moderate effects of triples excitations, ranging from -0.03 to $-0.11 \mathrm{eV}$. We expect that in these cases of moderate triples corrections, the differences between $\mathrm{CC} 3$ (or CCSDR(3)) and CCSD should give a rather accurate indications of the effects of higher excitations.

Except for the $2{ }^{1} E_{2 g}$ state-to which we shall return in the next section-we observe good convergence in the CC hierarchy. Thus, whereas the CCS errors relative to $\mathrm{CC} 3$ are large for valence excitations and the $2{ }^{1} E_{1 u}$ state (up to about $1 \mathrm{eV}), \mathrm{CC} 2$ reduces the error to less than $0.2 \mathrm{eV}$. For most Rydberg excitations, however, the CCS method gives remarkably good results. Indeed, for these excitations, CC2 does not represent any improvement on CCS, overshooting the doubles contributions compared to CCSD. This is in contrast to what is usually observed, and is likely to be due to fortuitous good results for CCS. The magnitude of the shift at each step in the hierarchy CCS, CC2, CCSD, CC3 are for all excitations except $2{ }^{1} E_{2 g}$ similar to recent benchmark calculations on several small molecules, where $\mathrm{CC} 3$ gave results within $0.05 \mathrm{eV}$ of the FCI results for excitations dominated by a single replacement (with a $t_{1}$ weight larger than $90 \%)$.

The $2{ }^{1} E_{2 g}$ excitation is rather special, having consider- able contributions from double replacements relative to the ground state. The double replacement character is not described at all in the CCS model, which is therefore completely in error for this excitation. In $\mathrm{CC} 2$, the double excitation part is only described to zero order in the ground state fluctuation potential. In CCSD, the description of the double replacement character is improved to first order, and for CC3 and the non-iterative triples methods second-order quality is obtained. Whereas large errors still persist for pure doublesreplacement dominated excitations, single-replacement dominated excitations with a significant double-replacement character are expected to be described reasonably well at the CCSD level.

For the $2{ }^{1} E_{2 g}$ excitation, the $t_{1}$ weight is $85 \%$ in CCSD and a reasonably accurate excitation energy should therefore be expected for this wave function. Nevertheless, we find that the effect of the triples is very large and that the $t_{1}$ weight is reduced to $66 \%$ in $\mathrm{CC} 3$. The different triples approaches give results ranging from 8.4 to $8.7 \mathrm{eV}$-that is, from 0.5 to $0.8 \mathrm{eV}$ from the CCSD result. Clearly, the large shift relative to CCSD and the variations in results obtained for the different triples approximations indicate that this state is not described as accurately as the other states. The CC2 and CCSD solutions for the $2{ }^{1} E_{2 g}$ excitation do not appear to be very different in character and the $t_{1}$ weight does not appear to be highly critical for an accurate CCSD description. In contrast, in for example $N_{2}$ we have found in recent FCI benchmark calculations ${ }^{47}$ that a state with a similar $t_{1}$ weight was described within an error of $0.4 \mathrm{eV}$ in CCSD, but with uniform convergence in the CCS, CC2, CCSD, and CC3 series. The large effect of higher excitations indicates that when considering systems with conjugated $\pi$ electron 
TABLE VII. $\mathrm{C}_{6} \mathrm{H}_{6}$ singlet excitation energies in $\mathrm{eV}$ from selected theoretical investigations.

\begin{tabular}{|c|c|c|c|c|c|c|c|c|c|c|c|}
\hline Method & $\begin{array}{l}\text { CAS- } \\
\mathrm{SCF}^{\mathrm{a}}\end{array}$ & $\begin{array}{l}\text { CAS- } \\
\text { PT2 }^{\text {a }}\end{array}$ & $\mathrm{RPA}^{\mathrm{b}}$ & SOPPA $^{b}$ & $\mathrm{MRMP}^{\mathrm{c}}$ & CISDT $^{\mathrm{d}}$ & $\mathrm{SAC}-\mathrm{CI}^{\mathrm{e}}$ & $\mathrm{MRCI}^{\mathrm{f}}$ & $\mathrm{SCVB}^{\mathrm{g}}$ & $\begin{array}{c}\text { CIS }(D)^{\mathrm{h}} \\
\text { (f.c.) }\end{array}$ & $\begin{array}{c}\operatorname{CCSD}^{\mathrm{h}} \\
\text { (f.c.) }\end{array}$ \\
\hline${ }^{1} B_{2 u}\left(V-\pi \pi^{*}\right)$ & 4.80 & 4.84 & 5.82 & 4.69 & 4.77 & 5.00 & 5.25 & 5.19 & 4.94 & 5.36 & 5.23 \\
\hline $1^{1} B_{1 u}$ & 7.32 & 6.30 & 5.88 & 6.01 & 6.28 & 7.64 & 6.60 & 7.20 & 7.49 & 6.76 & 6.68 \\
\hline $1{ }^{1} E_{1 u}$ & 8.53 & 7.03 & 7.16 & 6.75 & 6.98 & 8.34 & 7.47 & 8.16 & 8.12 & 7.41 & 7.47 \\
\hline $2{ }^{1} E_{2 g}$ & 7.96 & 7.90 & & & 7.88 & 8.33 & & 8.17 & 7.98 & & \\
\hline $2{ }^{1} E_{1 u}\left(R-\pi \pi^{*}\right)$ & 6.46 & 7.16 & 7.50 & 7.03 & 7.01 & 7.26 & 6.91 & 7.05 & 7.10 & & \\
\hline $2{ }^{1} A_{1 g}$ & 7.14 & 7.74 & 7.77 & 7.56 & 7.62 & 7.92 & 7.64 & 7.88 & 7.77 & & \\
\hline $1^{1} E_{2 g}$ & 7.09 & 7.77 & 7.80 & 7.55 & 7.63 & 7.90 & 7.64 & 7.90 & 7.76 & & \\
\hline $1^{1} A_{2 g}^{2 g}$ & 7.08 & 7.81 & 7.85 & 7.59 & 7.66 & 7.94 & 7.57 & 7.80 & 7.81 & & \\
\hline $1^{1} E_{1 g}\left(R-\pi \sigma^{*}\right)$ & 6.26 & 6.38 & 6.54 & 6.18 & 6.39 & & 6.31 & 6.32 & 6.33 & 6.87 & 6.84 \\
\hline $1^{1} A_{2 u}$ & 6.66 & 6.86 & 6.94 & 6.70 & 6.84 & & 6.88 & 6.69 & 6.86 & 7.33 & 7.32 \\
\hline $1{ }^{1} E_{2 u}$ & 6.74 & 6.91 & 7.11 & 6.76 & 6.92 & & 6.99 & 7.03 & 6.94 & 7.42 & 7.44 \\
\hline $1{ }^{1} A_{1 u}$ & 6.82 & 6.99 & 7.28 & 6.83 & 6.93 & & 7.10 & 7.23 & 7.04 & & \\
\hline $1{ }^{1} B_{2 g}$ & 7.33 & 7.58 & 7.68 & 7.35 & 7.53 & & 7.42 & & 7.55 & & \\
\hline $1{ }^{1} B_{1 g}$ & 7.29 & 7.58 & 7.70 & 7.35 & 7.51 & & 7.42 & & 7.51 & & \\
\hline $2{ }^{1} E_{1 g}$ & 7.33 & 7.57 & 7.59 & 7.34 & 7.56 & & 7.35 & & 7.56 & & \\
\hline $3{ }^{1} E_{1 g}$ & 7.37 & 7.57 & 7.73 & 7.40 & 7.61 & & 7.44 & & 7.48 & & \\
\hline
\end{tabular}

a Reference 56. $R_{\mathrm{CC}}=1.3950 \AA$ and $R_{\mathrm{CH}}=1.0850 \AA$. Bsis set is the ANO1 basis set described in footnotes for Table $\mathrm{V}$.

${ }^{\mathrm{b}}$ Reference 57. $R_{\mathrm{CC}}=1.3950 \AA$ and $R_{\mathrm{CH}}=1.0850 \AA$. Basis set from Ref. 56, the ANO1 basis set described in footnotes for Table V.

${ }^{\mathrm{c}}$ Reference 58. $R_{\mathrm{CC}}=1.397 \AA$ and $R_{\mathrm{CH}}=1.084 \AA$. Basis set is cc-pVDZ+CM[888/111] (123 basis functions).

${ }^{\mathrm{d}}$ Results from Table V in Ref. 52. $R_{\mathrm{CC}}=2.63662 \mathrm{bohr}$ and $R_{\mathrm{CH}}=2.05039$ bohr. Basis set is a C[95/42],H[4/2] augmented with $2 p \pi$ diffuse functions were added on each carbon. (108 basis functions).

${ }^{\mathrm{e}}$ Results from Tables V and IX in Ref. 54. $R_{\mathrm{CC}}=1.397 \AA$ and $R_{\mathrm{CH}}=1.084 \AA$. Basis set is an extension of the basis used in the CISDT calculations (basis 0) The result is for basis II for valence states and basis III for Rydberg states. Basis II=basis 0+ polarization functions: $\mathrm{C}(1 \mathrm{~d})$ ), $\mathrm{H}(1 \mathrm{p})$. (138 basis functions) Basis $\mathrm{III}=$ Basis $0+(2 s 2 p 2 d)$ on the center of mass (126 basis functions).

${ }^{\mathrm{f}}$ Reference 60. Hartree-Fock optimized geometries not given in Ref. 60. Results for basis B: C[95/42], H[4/2], CM[322/322]. (93 basis functions).

${ }^{g}$ Reference 59. Results from Tables XXIV and XXV in Ref. 59. $R_{\mathrm{CC}}=1.395 \AA$ and $R_{\mathrm{CH}}=1.085 \AA$. The present result correspond to Basis II in Ref. 54 for valence states and Basis III for Rydberg States. These are similar to the basis set of the SAC-CI studies.

${ }^{h}$ Result, Ref. 78. $R_{\mathrm{CC}}=1.395 \AA$ and $R_{\mathrm{CH}}=1.085 \AA$. Basis set is $6-31+\mathrm{G}^{*}$.

systems, one should be careful since connected higher excitations may be important for some states although this is not completely evident from the $t_{1}$ weight. The analogue to this state can be found in many organic molecules.

\section{Comparison with other theoretical studies}

In Table VII are given selected theoretical results for the excitation energies in benzene. The CASSCF and CASPT2 calculations of Ref. 56 and the RPA and SOPPA calculations of Ref. 57 have been carried out using the same ANO1 basis set and the same geometry as in our calculations and a direct comparison with these results is thus possible. For all excitations except the $2{ }^{1} E_{2 g}$ valence state, we expect as discussed in the previous section that the $\mathrm{CC} 3$ excitation energies are very close to the exact results in the same basis and at the same molecular geometry.

The CASSCF errors are large for the valence statesmore than $1 \mathrm{eV}$ for the ${ }^{1} E_{1 u}$ valence state. For the Rydberg $\pi$ - $\pi^{*}$ states, the CASSCF results are 0.8 to $1.0 \mathrm{eV}$ below the $\mathrm{CC} 3$ results. For the $\pi-\sigma^{*}$ states, they are 0.3 to $0.4 \mathrm{eV}$ below CC3. The CASPT2 correction reduce these errors significantly. Although the overall corrections to the total energies and to the excitation energies in CASPT2 are large, the excitation energies are within $0.3 \mathrm{eV}$ of the $\mathrm{CC} 3$ results. Thus, with CASPT2 corrections of up to $1.0-1.5 \mathrm{eV}$, the valence states are improved to be only $0.24\left(1{ }^{1} B_{2 u}\right), 0.24$ $\left(1^{1} B_{1 u}\right)$ and $0.1\left(1^{1} E_{1 u}\right)$ eV lower than CC3. For the Ryd- berg $\pi$ - $\pi^{*}$ excitation, we find that the $2{ }^{1} E_{1 u}$ state is $0.26 \mathrm{eV}$ below $\mathrm{CC} 3$, whereas for the other state the CASPT2 results is of order $0.1 \mathrm{eV}$ lower. Again we observe that the $2{ }^{1} E_{1 u}$ state behaves differently from the other Rydberg states. For the Rydberg $\pi-\sigma^{*}$ states, the CASPT2 results are typically $0.1 \mathrm{eV}$ below CC3. The CASPT2 excitation energies are consistently lower than the $\mathrm{CC} 3$ energies.

For the $2{ }^{1} E_{2 g}$ valence-state excitation, we observe a large difference between the CC and CASPT2 results: $8.4 \mathrm{eV}$ for $\mathrm{CC} 3$ versus $7.9 \mathrm{eV}$ for CASPT2. For this excitation, the CASSCF and CASPT2 results are in close agreement. This agreement does not imply that the CASPT2 results are accurate, however this excitation is difficult to describe in coupled cluster theory but presents no special problems for CASPT2. The large difference between CCSD and CC 3 suggests that the position of this excitation cannot be fully resolved in this study.

CASPT2 calculations have also been reported using another ANO basis and different active spaces. ${ }^{55}$ It is interesting to note that differences of up to $0.2 \mathrm{eV}$ are obtained in these CASPT2 calculations. Similar differences were also found in recent $\mathrm{CC} 2$ calculations using the same two ANO basis sets. ${ }^{51}$ However, there is little correspondence between the differences for the excitation energies obtained in the CASPT2 and in the CC2 calculations. We conclude that additivity of basis-set effects does not hold between CASPT2 
and coupled cluster theory and/or that the effect of using different active spaces is large.

The RPA results of Packer et al. are of the same quality as the CCS results (the $2{ }^{1} E_{1 u}$ state is again an exception). ${ }^{57}$ Note that no results are given for the $2{ }^{1} E_{2 g}$ state since it is not included in the investigation of Packer et al., probably because of the large contributions from double excitations. The SOPPA excitation energies are lower than the CC energies for all excitations. Note that $\mathrm{CC} 2$ is too low relative to CC3 for all Rydberg states and that SOPPA is still lower for these states. For these states, the difference between SOPPA and $\mathrm{CC} 3$ is $0.3 \mathrm{eV}$ and between CC2 and SOPPA of the order $0.2 \mathrm{eV}$. For the valence states, the difference between SOPPA and CC 3 is of order 0.4 to $0.5 \mathrm{eV}$. There is no parallel behavior of the $\mathrm{CC} 2$ and SOPPA results.

In another recent investigation, Hirao et al. have presented benzene excitation energies using their multireference Møller-Plesset perturbation theory. ${ }^{58}$ No direct comparison is possible since these workers employ a geometry as well as a basis set (cc- $p$ VDZ augmented with Rydberg functions) slightly different from ours. The results of Hirao et al. are within $0.12 \mathrm{eV}$ of the CASPT2 results.

Given in Table VII are also the configuration interaction (CI) calculations with singles, doubles and triples excitations (with selected configurations) of Hay and Shavitt from 1973 (Ref. 52) as well as results from symmetry-adapted cluster $\mathrm{CI}$ (SAC-CI). ${ }^{54}$ At a formal level, the excitation energies in $\mathrm{SAC}-\mathrm{CI}$ are equivalent to $\mathrm{CC}$ linear response energies. However, due to the introduction of various approximations, the resulting SAC-CI approach is significantly different from the coupled cluster linear response method. Also listed are the multireference CI (MRCI) results of Palmer and Walker and spin-coupled valence-bond results of da Silva et al. ${ }^{59}$ In spite of the small basis sets (relative to present standard), remarkably accurate excitation energies are obtained for the Rydberg states, whereas the results for the valence excitations are less accurate as is evident from Table VII.

A preliminary set of CCSD excitation-energy calculations on benzene has been reported by Head-Gordon et al. ${ }^{78}$ using a $6-31+G^{*}$ basis set and frozen core orbitals. Results from CI singles CIS (equivalent to CCS for excitation energies) and CIS with a perturbative doubles correction CIS(D) were also reported in this paper. The CIS(D) and CCSD excitation energies are given in Table VII. Comparing these energies with ours in Tables V and VI, it is evident that this basis set gives errors of order $0.3 \mathrm{eV}$. As stated by HeadGordon et al., these calculations should be considered as preliminary. CIS(D) and CC2 are both correct to second order in the ground-state fluctuation potential for a singlereplacement dominated excitation. Accordingly, we find that the difference between CIS(D) and CCSD to be of the same order of magnitude as the difference between $\mathrm{CC} 2$ and CCSD.

\section{E. Comparison with experiment}

Numerous experimental investigations of the benzene spectrum have been reported. In our calculations and those reported by others, only vertical excitation energies have been computed. A comparison with the experimental $0-0$ origin transitions requires that geometry relaxation and zeropoint vibrations are taken into account. For high-symmetry molecules like benzene, Jahn-Teller effects are also important. ${ }^{79,80}$

In this study, we have not investigated the geometry and vibrational effects in detail. Many effects thus still remain to be accounted for in order to give a detailed comparison with experiment and to offer a definite theoretical description of the complete benzene spectrum, including the vibronic structure and so on. In the following, we briefly review the experimental results in order to emphasize the assumptions that must be made in comparisons with the theoretical results and the limitations of such comparisons.

In Table VIII, we give origins for the benzene transitions when these have been assigned. Also, we give in some cases the value for the vibrational transition with maximum frequency or simply the maximum in the cases where the vibrational structure has not been resolved. Although we may not interpret the maximum transitions as energy differences between the potential energy-surfaces in the BornOppenheimer picture, the difference between energies at the Franck-Condon maximum and the origin may give an estimate of geometry relaxation effects.

\section{Valence states}

The valence states of benzene have most recently been observed by Hiraya and Shobatake in direct absorption spectra of jet-cooled benzene. ${ }^{73}$ The ${ }^{1} B_{2 u}$ valence state is observed with an origin at $4.787 \mathrm{eV}$ and a maximum at 4.902 $\mathrm{eV}$. These results are in excellent agreement with earlier electron-impact spectra of Lasserte et al. ${ }^{63}$ (4.790 (O), 4.902 $(\max ))$ and earlier ultraviolet absorption spectra.

The ${ }^{1} B_{1 u}$ valence state is observed by Hiraya and Shobatake to have an origin at $6.0348 \mathrm{eV}$ and maximum at 6.204 $\mathrm{eV}$. In this area, a rather diffuse vibrational progression is observed in electron-impact spectra. Lasserte $e t$ al. argue that two states contributes-the one with a peak at $6.2 \mathrm{eV}$ and the other with bands at $6.31,6.41,6.53 \mathrm{eV}$-but no term symbol was assigned for these states. The observed region agrees with the ${ }^{1} B_{1 u}$ maximum band and a series originating from the $1{ }^{1} E_{1 g}$ Rydberg state discussed later. Bands similar to the one of Lasserte et al. is observed in the absorption spectrum by Koch and Otto. ${ }^{64}$

The $1{ }^{1} E_{1 u}$ valence state has been observed in many spectra. In the absorption spectra of Koch and Otto, a maximum peak is observed at $6.94 \mathrm{eV}$, which is probably due to the $1^{1} E_{1 u}$ valence state. This transition agrees well with what was observed by Wilkinson. ${ }^{62}$ Hiraya and Shobatake gives an origin for this transition at $6.866 \mathrm{eV}$.

For all these states, we have differences between the 0-0 transition and the maximum-intensity transition of about 0.1 to $0.2 \mathrm{eV}$. A preliminary CCSD calculation using the ${ }^{1} B_{2 u}$ experimental geometry ${ }^{81}\left(R_{\mathrm{CC}}=1.434 \AA, R_{\mathrm{CH}}=1.07 \AA\right)$ gave a difference between the vertical and the adiabatic transitions of $0.214 \mathrm{eV}$. Taking the large basis-set CCSD results and including this correction and corrections for the effect of 
TABLE VIII. $\mathrm{C}_{6} \mathrm{H}_{6}$ singlet excitation energies in eV. CCSD excitation energies and triples corrections. Experimental origins and maxima.

\begin{tabular}{|c|c|c|c|c|}
\hline Method & $\begin{array}{c}\Delta E \\
\operatorname{CCSD}^{\mathrm{a}}\end{array}$ & $\begin{array}{c}\Delta E_{\text {Triples }} \\
\text { (f.c. CC3- } \\
\text { CCSD ANO1) }\end{array}$ & $\begin{array}{c}\Delta E \\
\text { Origin }\end{array}$ & $\begin{array}{c}\Delta E \\
\text { Maximum }\end{array}$ \\
\hline${ }^{1}{ }^{1} B_{2 u}\left(e_{1 g} \rightarrow e_{2 u}\right)$ & 5.180 & -0.111 & $4.7873,^{\mathrm{b}} 4.790^{\mathrm{c}}$ & $4.902^{\mathrm{b}, \mathrm{c}}$ \\
\hline${ }^{1} B_{1 u}\left(e_{1 g} \rightarrow e_{2 u}\right)$ & 6.481 & -0.050 & $6.0348^{\mathrm{b}}$ & $6.204^{\mathrm{b}}$ \\
\hline $1^{1} E_{1 u}\left(e_{1 g} \rightarrow e_{2 u}\right)$ & 7.227 & -0.034 & $6.8656^{\mathrm{b}}$ & $6.95,{ }^{\mathrm{c}} 6.94,{ }^{\mathrm{d}} 6.96^{\mathrm{e}}$ \\
\hline $2{ }^{1} E_{2 g}\left(a_{2 u} \rightarrow e_{2 u}\right)$ & 9.168 & -0.765 & & $7.8^{f}$ \\
\hline $2{ }^{1} E_{1 u}\left(e_{1 g} \rightarrow 3 p_{0}\right)$ & 7.501 & -0.159 & $7.413^{\mathrm{e}}$ & same \\
\hline $2{ }^{1} A_{1 g}\left(e_{1 g} \rightarrow 3 d_{1}\right)$ & 7.984 & 0.010 & $7.807,{ }^{\mathrm{i}} 7.808,{ }^{\mathrm{j}} 7.819^{\mathrm{k}}$ & same \\
\hline $1^{1} E_{2 g}\left(e_{1 g} \rightarrow 3 d_{1}\right)$ & 7.972 & 0.009 & $7.805^{\mathrm{i}}$ & same \\
\hline $1{ }^{1} A_{2 g}\left(e_{1 g} \rightarrow 3 d_{1}\right)$ & 8.009 & 0.006 & - & - \\
\hline${ }^{1} E_{1 g}\left(e_{1 g} \rightarrow 3 s\right)$ & 6.563 & -0.028 & $6.334^{\mathrm{g}}$ & same \\
\hline $1{ }^{1} A_{2 u}\left(e_{1 g} \rightarrow 3 p_{1}\right)$ & 7.092 & -0.008 & $6.932,{ }^{\mathrm{h}} 6.928^{\mathrm{b}, \mathrm{e}}$ & same \\
\hline${ }^{1} E_{2 u}\left(e_{1 g} \rightarrow 3 p_{1}\right)$ & 7.169 & -0.018 & $6.953^{\mathrm{h}}$ & same \\
\hline $1{ }^{1} A_{1 u}\left(e_{1 g} \rightarrow 3 p_{1}\right)$ & 7.262 & -0.024 & - & - \\
\hline $1^{1} B_{2 g}\left(e_{1 g} \rightarrow 3 d_{2}\right)$ & 7.760 & -0.003 & - & - \\
\hline${ }^{1} B_{1 g}\left(e_{1 g} \rightarrow 3 d_{2}\right)$ & 7.751 & -0.005 & - & - \\
\hline $2{ }^{1} E_{1 g}\left(e_{1 g} \rightarrow 3 d_{0}\right)$ & 7.671 & 0.001 & $7.535,{ }^{i} 7.540^{j}$ & same \\
\hline $3{ }^{1} E_{1 g}\left(e_{1 g} \rightarrow 3 d_{2}\right)$ & 7.772 & -0.005 & - & - \\
\hline $3{ }^{1} E_{2 g}\left(\sigma e_{1 g} \rightarrow 3 s\right)$ & 9.387 & -0.132 & $8.552,{ }^{\mathrm{k}} 8.564^{\mathrm{j}}$ & - \\
\hline${ }^{1} E_{2 g}\left(e_{1 g} \rightarrow 4 d_{1}\right)$ & 8.591 & & $8.437^{\mathrm{k}}$ & same \\
\hline${ }^{1} A_{1 g}\left(e_{1 g} \rightarrow 4 d_{1}\right)$ & 8.599 & & $8.440,{ }^{\mathrm{k}} 8.440,{ }^{\mathrm{i}} 8.442^{\mathrm{j}}$ & same \\
\hline${ }^{1} A_{2 g}\left(e_{1 g} \rightarrow 4 d_{1}\right)$ & 8.605 & & - & - \\
\hline
\end{tabular}

${ }^{\text {aSee footnotes to Tables V and VI for detail on calculations. Conversion factor used in translating experimental }}$ results in $\mathrm{cm}^{-1}$ to $\mathrm{eV}$ is 8065.5409 .

${ }^{\mathrm{b}}$ Reference 73 .

${ }^{\mathrm{c}}$ Reference 63.

${ }^{\mathrm{d}}$ Reference 64.

${ }^{\mathrm{e}}$ Reference 62.

${ }^{\mathrm{f}}$ References $67-68$.

${ }^{\mathrm{g}}$ References 65

${ }^{\mathrm{h}}$ References 66.

${ }^{\mathrm{i}}$ References 69

${ }^{\mathrm{j}}$ Reference 72.

${ }^{\mathrm{k}}$ Reference 71 .

triples, we arrive at an estimate of $4.855 \mathrm{eV}$ for the adiabatic transition. Using the incomplete set of frequencies given by Parmenter for the ground state and for the ${ }^{1} B_{2 u}$ state, ${ }^{82}$ we obtain a difference in zero-point energy of about $0.06 \mathrm{eV}$, considering only the modes where data are given for the ${ }^{1} B_{2 u}$ state (half of the modes). This correction brings the result even closer to the $4.787 \mathrm{eV}$ origin.

It should be emphasized that these estimates are rather crude. However, it is clear that before entering into geometrical considerations we cannot hope for better agreement than the one in Table VIII. Although the above estimates are preliminary, it appears probable that geometry and zero-point vibrational effects should account for a large fraction of the difference between the 0-0 transitions and the coupled cluster vertical excitation energies. Most significantly, it appears to be more important to take into consideration geometrical effects than to go beyond CCSD for these excited states.

In laser flash experiments of Nakashima et al., three peaks were observed and interpreted as states approximately $7.0 \mathrm{eV}, 7.8 \mathrm{eV}$ and $9.4 \mathrm{eV}$ above the ground state. ${ }^{67,68}$ The excitation energies from the ground state are obtained by addition of the observed transition from the ${ }^{1} B_{2 u}$ state and a value of $4.72 \mathrm{eV}$ for the ground state to ${ }^{1} B_{2 u}$ state $(0-0$ transition from Ref. 82 in cyclohexane solution). Use of newer values for the ${ }^{1} A_{1 g}$ to ${ }^{1} B_{2 u}$ excitation may give a small increase in the magnitudes of the excitation energies as seen from the numbers in Table VIII.

The first state has been assigned ${ }^{1} E_{1 u}$, in reasonably agreement with the observations cited above. Lorentzon et al. and others choose to assign the second state as $2{ }^{1} E_{2 g}$, based on the suggestion that the $1{ }^{1} E_{2 g}$ state is very diffuse and the transition to this state should be considerably less intense than the excitation to the valence state $2{ }^{1} E_{2 g} .{ }^{56}$ If the $7.8 \mathrm{eV}$ peak does represent the $2{ }^{1} E_{2 g}$ valence state, it may still contain contributions from the $1{ }^{1} E_{2 g}$ Rydberg state. These assignments leave us with the problem of interpreting the $9.4 \mathrm{eV}$ peak. We find it difficult to agree on an excitation energy of $7.8 \mathrm{eV}$ but neither can we conclude that the $9.4 \mathrm{eV}$ peak corresponds to the $2{ }^{1} E_{2 g}$ valence state. Triples are certainly important for this state and the neglect of triples could lead to an incorrect assignment. Furthermore, we have additional uncertainties since geometry and vibration effects in all three states may play a role. 


\section{Rydberg states}

We consider in this study mainly the first members $(n$ $=3$ ) of the Rydberg series, which converges to the first ionization potential of $9.246 \mathrm{eV} .{ }^{70}$ Since the pioneering work of Price and Wood ${ }^{61}$ and Wilkinson ${ }^{62}$ many experimental studies of the Rydberg states of benzene have been reported. Only few transitions are dipole-allowed but many states have been observed in multiphoton ionization (MPI) spectroscopy. Jahn-Teller effects may complicate the assignments.

The Jahn-Teller splitting in benzene is interesting since it contains splittings originating both from the degenerate core and from the Rydberg electron, which may or may not be in a degenerate orbital. ${ }^{79,80}$ Raghavachari et al. ${ }^{83}$ and Hirao and Kato ${ }^{84}$ have calculated the Jahn-Teller stabilization of the benzene ${ }^{2} E_{1 g}$ cation to be $0.12-0.13 \mathrm{eV}$ by UHFbased geometry optimizations and subsequent MP2 and SAC-CI energy calculations, respectively. In both cases, the basis sets are rather small $(6-31 \mathrm{G}$ and 4-31G) and the resulting energy differences cannot be expected to be accurate. The HF stabilization was found to be $0.15 \mathrm{eV} .^{83}$ The JahnTeller effect in the Rydberg states is the same as in the ioncore in the limit of large $n$. Assuming in a first approximation that this Jahn-Teller stabilization carries over to the Rydberg states of benzene, the vertical excitations energies should be 0.1 to $0.15 \mathrm{eV}$ too high. Preliminary calculations were carried out using the Jahn-Teller split benzene-cation ${ }^{2} B_{2 g}$ 6-31G UHF geometry of Ref. 83 in a CCSD aug$p$ VDZ-CM2 excitation-energy calculation. We obtain a shift between the vertical excitation energies and the excitation from the ground-state equilibrium geometry to the point on the adiabatic Rydberg excited state surfaces of order 0.10 $\pm 0.03 \mathrm{eV}$ for all Rydberg states (again with the $1{ }^{1} E_{1 u}$ as the exception). The same shift was observed for the $n=3$ and $n=4\left(d_{1}\right)$ Rydberg states indicating the common origin of this shift in the cation core. Again these estimates are rather crude, however an order of magnitude is obtained.

In the following, we shall restrict our comparisons to listing the experimental 0-0 transitions and comparing these with our vertical excitation energies, keeping the above estimates of other effects in mind. A detailed analysis of JahnTeller effects is left for future investigations. Rydberg states higher than $n=3$ are generally not treated in this study but are discussed briefly in a separate section. The first state of $E_{2 g}$ symmetry originating from the Rydberg series converging to the second ionization potential is also briefly discussed in a separate section.

a. $3 s$ Rydberg states: The $1{ }^{1} E_{1 g}$ Rydberg state was first observed by Johnson as a two-photon resonance in the three-photon ionization region of benzene with origin at $6.334 \mathrm{eV}$, and with a band structure in the region 6.3 to 6.6 $\mathrm{eV} .{ }^{65}$ At the time of the experiment it was not clear whether this state should be assigned as the ${ }^{1} E_{2 g}$ valence or as a Rydberg $(3 s)^{1} E_{1 g}$ state. It has later been ruled out that this state is the valence ${ }^{1} E_{2 g}$ state. ${ }^{67,68,85}$ This assignment is supported by our calculations as well as those of others. Our vertical excitation energy is $0.2 \mathrm{eV}$ larger than the experimental origin. b. $3 p$ Rydberg states: The excitation of an electron to a degenerate $3 p_{1}\left(p_{x . y}\right.$ or $3 p_{\sigma}$ ) orbital gives rise to ${ }^{1} A_{1 u},{ }^{1} A_{2 u}$, and ${ }^{1} E_{2 u}$ states. The $1^{1} A_{2 u}\left(3 p_{1}\right)$ Rydberg state is dipoleallowed and has been observed in several spectra since the pioneering work of Wilkinson $(6.928 \mathrm{eV}(\mathrm{O})) .^{62} \mathrm{Johnson}$ and Korenowski obtain the origin at $6.932 \mathrm{eV}$ in their threephoton MPI experiment. ${ }^{66}$ In their three-photon spectrum, Johnson and Korenowski also identified the $1{ }^{1} E_{2 u}\left(3 p_{1}\right)$ Rydberg state with origin at $6.953 \mathrm{eV}$. The third $3 p_{\sigma}$ Rydberg state, $1{ }^{1} A_{1 u}$, has to our knowledge not been observed. The assignment of vibronic structure of these transitions has been somewhat controversial and a correct explanation requires consideration of the Jahn-Teller effect. ${ }^{79,80}$

Since the triples effects are small for the Rydberg states, we expect the discrepancies between the calculated results and the experimental $0-0$ transitions to arise from JahnTeller and vibrational effects. Thus, these corrections are in the range 0.1 to $0.2 \mathrm{eV}$ in agreement with the estimates discussed previously. The order of the states is predicted in the CC vertical energy calculations to be ${ }^{1} A_{2 u},{ }^{1} E_{2 u}$, and ${ }^{1} A_{1 u}$. This order agrees with the above cited experimental 0-0 transition where the ${ }^{1} A_{2 u}$ is below the ${ }^{1} E_{2 u}$ state. Earlier results had a reversed ordering, see Ref. 80 for a discussion. Whetten and Grant have in their analysis of Jahn-Teller effects assumed that the ${ }^{1} A_{2 u}$ and ${ }^{1} A_{1 u}$ states are at similar energies and below ${ }^{1} E_{2 u}$, which is in contrast with our results. ${ }^{80}$ In the recent work of Staib and Domcke on the Jahn-Teller effects in benzene, ${ }^{79}$ the ordering of states is the same as ours. All ab initio investigations in Table VII agree on this matter.

The $3 p_{\pi}$ Rydberg state is the dipole-allowed $1{ }^{1} E_{1 u}$ state. Wilkinson observed this state with an origin at 7.413 $\mathrm{eV}^{62}$ This is the most intense $0-0$ transition. As already noted, this state behaves differently from the other Rydberg states-the triples corrections are larger and the basis effects different. Just as the valence ${ }^{1} E_{1 u}$ state at $7.2 \mathrm{eV}$ is known to be relatively diffuse (see for example Lorentzon et al.), this state may have a mixed valence-Rydberg character. Furthermore, whereas our vertical excitation energies for the Rydberg states usually lie between 0.1 and $0.2 \mathrm{eV}$ above the spectroscopic 0-0 transitions, this is not the case for this state (including triples corrections). This agrees with our preliminary calculations on Jahn-Teller effects described above where no Jahn-Teller stabilization was found for this state in contrast to the other Rydberg states.

c. $3 d$ Rydberg states: The Rydberg $d$ orbitals in $D_{6 h}$ contributes to orbitals of $a_{1 g}, e_{1 g}$, and $e_{2 g}$ symmetries. Exciting an $e_{1 g}$ electron into the $3 d_{1}$ (or $3 d_{x z, y z}$ or $3 d_{\pi}$ ) orbitals gives rise to states of symmetries ${ }^{1} A_{1 g},{ }^{1} A_{2 g}$ and ${ }^{1} E_{2 g}$. The excitation into $3 d_{0}$ (or $3 d_{\sigma}$ ) gives a state of ${ }^{1} E_{1 g}$ symmetry, and excitation to $3 d_{2}$ (or $3 d_{\delta}$ ) gives rise to states of ${ }^{1} B_{1 g}$, ${ }^{1} B_{2 g}$ and ${ }^{1} E_{1 g}$ symmetry. States of ${ }^{1} A_{1 g},{ }^{1} E_{1 g}$ and ${ }^{1} E_{2 g}$ symmetry are two-photon allowed.

Whetten, Fu, and Grant observed the first member of a gerade Rydberg series with origin at $7.807 \mathrm{eV} .{ }^{69}$ In polarized two-photon spectroscopy, Whetten et al. observed both $E$ and $A$ symmetry states with origins at 7.805 and $7.807 \mathrm{eV}$, respectively. Grubb et al. reports an origin at $7.819 \mathrm{eV}$, and 
argues that contributions from three-photon ${ }^{1} B_{1 u}$ intermediates may be present in the four-photon spectrum. Thus, the most reliable assignment probably is the ${ }^{1} E_{2 g}\left(3 d_{1}\right)$ origin at $7.805 \mathrm{eV}$ and the ${ }^{1} A_{1 g}\left(3 d_{1}\right)$ origin at $7.807 \mathrm{eV}$. Our vertical excitation energies are about $0.18 \mathrm{eV}$ higher than these.

Whetten, $\mathrm{Fu}$ and Grant observed an origin at $7.535 \mathrm{eV}$ in two-photon spectroscopy. ${ }^{69}$ It was suggested that the band should be assigned as the valence ${ }^{1} E_{2 g}$ state. It was later ruled out that this was the valence ${ }^{1} E_{2 g}$ state, and the energy fits well with either the ${ }^{1} E_{1 g}\left(3 d_{\sigma}\right)$ or the ${ }^{1} E_{1 g}\left(3 d_{\delta}\right)$ Rydberg states. Grubb et al. observe a state with origin at $7.540 \mathrm{eV}$ in four-photon resonant five-photon ionization spectroscopy. ${ }^{72}$ The authors argue - by analysis of the polarization behavior and quantum defects in relations to higher Rydberg statesthat this peak corresponds to the $3 d_{0}$ Rydberg state. We find some support of this in our calculations. Assigning the 7.54 $\mathrm{eV}$ peak to $2{ }^{1} E_{1 g}\left(3 d_{\sigma}\right)$, we obtain a discrepancy of 0.13 $\mathrm{eV}$ - that is, of the same magnitude as for most of the Rydberg states. If instead we interpret this peak as the $3{ }^{1} E_{1 g}\left(3 d_{\delta}\right)$ state-as done in several theoretical studies - we find a relatively large deviation of $0.23 \mathrm{eV}$.

Accepting this assignment, we find that transitions to the $3 d_{\delta}$ Rydberg states have not been observed directly. Transitions to the ${ }^{1} B_{1 g}$ and ${ }^{1} B_{2 g}$ states are first allowed in fourphoton transitions from the ground state. However, the higher-order components of these states have presumably been observed in the investigations of Grubb et al. as the so-called $R_{g}(0.24)$ Rydberg series. Using the empirically determined quantum defect, the $n=3$ member of this series is extrapolated to appear at about $7.449 \mathrm{eV}$. Grubb et al. thus propose this as a common estimate of the origin. Grubb et al. emphasize that a similar extrapolation of the $3 s$ Rydberg state from a quantum-defect fit to the higher Rydberg members, overestimates the observed value by $0.18 \mathrm{eV}$. We find that our results for the ${ }^{1} B_{1 g},{ }^{1} B_{2 g}$ and $3{ }^{1} E_{1 g} 3 d_{\delta}$ states are very similar, but $0.3 \mathrm{eV}$ above the estimated value. It is plausible that we should have a discrepancy of this magnitude due to the addition of the usual $0.15 \mathrm{eV}$ and an error in the estimate of same magnitude, although the error of the estimate is of opposite sign relative to the above cited.

d. $4 d_{1}$ Rydberg states: Higher Rydberg states can be observed in many spectra. In particular, for the allowed $p$-type Rydberg series there are many results. Most of the information for the gerade states comes from the extensive studies by Whetten and Grubb and co-workers. ${ }^{69-72}$ Our purpose is not to follow every Rydberg series to the limit, so we shall consider only the $n=4$ components of the $4 d_{1}$ series. Origins are observed at $8.437 \mathrm{eV}\left({ }^{1} E_{2 g}\right)$ and $8.440 \mathrm{eV}$ $\left({ }^{1} A_{1 g}\right)$. As for the $n=3$ series we find that our calculated vertical excitation energies are about $0.15 \mathrm{eV}$ higher than the experimental origins. The similarities in the deviations between our calculated vertical excitation energies and the observed origin for $n=3$ and $n=4$ Rydberg states strongly indicate a common explanation. In other words, the description of the ion core is responsibly for the $0.15 \mathrm{eV}$ discrepancy. As noted in the beginning of the Rydberg section, this may well be due to Jahn-Teller effects, which are calculated to be of that order of magnitude. e. $(3 s) E_{2 g}$ state: Whetten et al. observe a structure with origin at $8.552 \mathrm{eV},{ }^{71}$ and similarly Grubb et al. find an origin at $8.564 \mathrm{eV}^{72}$ Whetten, Grubb, and coworkers propose that this is the first member of a series converging to the first excited ionic state ${ }^{2} E_{2 g}$. This assignment is based on resemblances between the vibronic structures of the ${ }^{2} E_{2 g}$ photoelectron system and the vibronic structure of the Rydberg state. These values do not fit well with our vertical CCSD excitation energies of $9.38 \mathrm{eV}$. The effect of triples is slightly larger than for the other Rydberg states but only $-0.13 \mathrm{eV}$. It is difficult to believe that our vertical excitation energies are wrong by $0.8 \mathrm{eV}$ for this state. We conclude that either large geometrical effects are involved in this transition or that the assignment is incorrect.

\section{CONCLUDING REMARKS}

A long-standing goal of molecular quantum chemistry is to be able to carry out calculations that are accurate enough to explain and predict spectroscopic properties. In order to reach this goal, we must develop useful methods that allow us to estimate the accuracy of the theoretical calculations. In particular, we must be able to carry out systematic investigations of the accuracy with respect to the two basic approximations made in the solution of the electronic problem-the use of finite one-electron basis sets and the use of approximate $N$-electron models. In direct comparison with experiment, other effects must also be taken into account. For electronic spectra, geometrical and vibrational effects for the ground and excited states are particularly important. Calculations of oscillator strengths, vibrational frequencies of the ground and excited states, adiabatic transitions and so on are required for a more complete and useful comparison with experiment. However, before embarking on such an enterprise, it is important to address the fundamental theoretical requirements for obtaining an accurate electronic description of transition properties.

Recently, we have proposed a hierarchy of coupled cluster models and thoroughly tested their performance with encouraging results. In this paper, we have demonstrated how these models may be used in integral-direct calculations in large basis sets for calculation of excitation energies. The combination of integral-direct techniques with a hierarchy of coupled cluster models constitutes a very powerful tool for systematically addressing the fundamental requirement for accurate calculations of excitation energies. We have in this paper and in our previous paper on CC2 excitation energies systematically investigated basis-set and correlation effects in calculations of the vertical electronic excitation energies of benzene. We have investigated the basis-set convergence carefully by carrying out calculations with up to 432 basis functions. We have shown that to obtain an accuracy better than $0.2 \mathrm{eV}$ in $\mathrm{CC} 2$ and CCSD, polarized triple-zeta basis sets are required for the Rydberg excitations in addition to diffuse functions. The inclusion of triple excitations using CC3 and CCSDR(3) leads to even more accurate results and also provides us with an indication of the importance of higher excitations. In this study, we have found that triples- 
with one notable exception-give rather small corrections and conclude that the CCSD and CC3 results for these states are highly accurate. For these states, we may therefore assume that we have solved the electronic problem to a high accuracy relative to the remaining geometrical and vibrational corrections.

Only systematic investigations can support us in our conjecture that the remaining discrepancies with respect to experimental results is not due to errors in our approach for solving the electronic problem but to a large extent due to effects not treated in this study. Treating other than the electronic degrees of freedom in detail is beyond the scope of this investigation as it has been for previous theoretical investigations on benzene. Work is underway for taking geometrical and vibrational effects for both ground and excited states into account along the lines of Stanton and Gauss. ${ }^{90,91}$ Previous theoretical studies have to a large extent comprised significant errors with respect to both basis set and correlation as well as geometrical and vibrational effects is not taken into account. In many cases cancellation of errors and neglect of physical effects leads to fortuitous good agreement between calculated vertical excitation energies and experimental results. In our study we see very clearly the limitations of calculating only vertical excitation energies. It should also be emphasized that all effects (electron correlation, basis set, geometry relaxation, vibration etc.) may be larger in many other systems. Thus a proper theoretical description of all these effects are needed for complete description of electronic spectra.

Taking CC3 as a good approximation to the exact result within a given orbital basis for states with a $t_{1}$ weight larger than $90 \%$, we find that many popular theoretical approaches may easily contain errors in the benzene excitation energies of 0.2 to $0.3 \mathrm{eV}$ or even larger. On the other hand, for states such as $2{ }^{1} E_{2 g}$, the coupled cluster based methods certainly have their own problems. In our opinion, it is difficult to claim an accuracy better than 0.3 to $0.5 \mathrm{eV}$ for methods such as CC2, SOPPA, and CASPT2 in a polarized double-zeta basis augmented with diffuse functions. To go beyond this level of accuracy, many effects must be considered. For more accurate calculations, it is necessary to use more accurate $N$-electron wave functions and larger one-electron basis sets as done in this paper.

We would like to emphasize that for systematic and reliable studies of molecular electronic spectra and also for a meaningful comparison with experiment, high accuracy is required in the wave functions. We must have at our disposal some means of investigating the accuracy of our calculations both with respect to the truncations in the one-electron basis sets and with respect to the approximations in the $N$-electron space. We achieve this goal through the twin adoption of the hierarchy of correlation-consistent basis sets and the hierarchy of coupled cluster wave functions. In addition to demonstrating the feasibility of such large-scale coupled cluster calculations for excitation energies, we have shown that high accuracy and systematic calculations are of crucial importance in order to distinguish between real physical effects and errors arising from approximations made in the one- and
$N$-particle spaces. Presently our methods is unrivalled by any other $a b$ initio method in both accuracy and reliability. We therefore believe that the methods used in this paper provides valuable tools for reliable assignment of spectra for large molecules.

\section{ACKNOWLEDGMENTS}

This work has been partly supported by the Danish Natural Research Council (Grant No. 11-0924) and the Spanish CICYT project PB94-0993. We thank Berta Fernandez for critical reading of the manuscript, and Erik K. Dalskov for a preprint of Ref. 57 .

${ }^{1}$ C. W. Bauschlicher, Jr., S. R. Langhoff, and P. R. Taylor, Adv. Chem. Phys. 77, 103 (1990).

${ }^{2}$ For a review, see K. Anderson and B. O. Roos, in Modern Electronic Structure Theory, edited by D. Yarkoni (VCH, Weinhem, 1995).

${ }^{3}$ K. Anderson, P.-A. Malmqvist, B. O. Roos, A. J. Sadlej, and K. Wolinski, J. Phys. Chem. 94, 5483 (1990).

${ }^{4}$ K. Anderson, P.-A. Malmqvist, and B. O. Roos, J. Chem. Phys. 96, 1218 (1992).

${ }^{5}$ For a review, see J. Olsen and P. Jørgensen, in Modern Electronic Structure Theory, edited by D. Yarkoni (VCH, Weinhem, 1995).

${ }^{6}$ E. Nielsen, P. Jørgensen, and J. Oddershede, J. Chem. Phys. 73, 6238 (1980).

${ }^{7}$ For a review, see J. Oddershede, Adv. Chem. Phys. 69, 201 (1987).

${ }^{8}$ H. J. Monkhorst, Int. J. Quantum Chem. S11, 421 (1977).

${ }^{9}$ D. Mukherjee and P. K. Mukherjee, Chem. Phys. 39, 325 (1979).

${ }^{10}$ S. Gosh, D. Mukherjee, and D. Bhattacheryya, Chem. Phys. 72, 161 (1982).

${ }^{11}$ E. Dalgaard and H. J. Monkhorst, Phys. Rev. A 28, 1217 (1983).

${ }^{12}$ H. Koch and P. Jørgensen, J. Chem. Phys. 93, 3333 (1990).

${ }^{13}$ B. Datta, P. Sen, and D. Mukherjee, J. Phys. Chem. 99, 6441 (1995).

${ }^{14}$ O. Christiansen, H. Koch, and P. Jørgensen, J. Chem. Phys. 103, 7429 (1995).

${ }^{15}$ O. Christiansen, H. Koch, and P. Jørgensen, Chem. Phys. Lett. 243, 409 (1995).

${ }^{16}$ J. Olsen, P. Jørgensen, H. Koch, A. Balkova, and R. J. Bartlett, J. Chem. Phys. 104, 8007 (1996).

${ }^{17}$ P.- A. Malmqvist and B. O. Roos, Chem. Phys. Lett. 155, 189 (1989).

${ }^{18}$ J. Cizek, J. Chem. Phys. 45, 4256 (1966).

${ }^{19}$ J. Cizek and J. Paldus, Int. J. Quantum Chem. 5, 359 (1971).

${ }^{20}$ J. Paldus, J. Cizek, and I. Shavitt, Phys. Rev. A 5, 50 (1972).

${ }^{21}$ G. P. Purvis and R. J. Bartlett, J. Chem. Phys. 76, 1910 (1982).

${ }^{22}$ T. J. Lee and J. E. Rice, Chem. Phys. Lett. 150, 406 (1988).

${ }^{23}$ G. E. Scuseria, C. L. Jansen, and H. F. Schaefer, J. Chem. Phys. 89, 7382 (1988).

${ }^{24}$ J. F. Stanton, J. Gauss, J. D. Watts, and R. J. Bartlett, J. Chem. Phys. 94, 4334 (1991).

${ }^{25}$ C. Hampel, K. A. Peterson, and H.-J. Werner, Chem. Phys. Lett. 190, 1 (1992)

${ }^{26}$ H. Koch, O. Christiansen, R. Kobayashi, P. Jørgensen, and T. Helgaker, Chem. Phys. Lett. 228, 233 (1994).

${ }^{27}$ H. Koch, A. Sanchez de Meras, T. Helgaker, and O. Christiansen, J. Chem. Phys. 104, 4157 (1996).

${ }^{28}$ G. Fitzgerald, R. J. Harrison, W. D. Laidig, and R. J. Bartlett, Chem. Phys. Lett. 117, 433 (1985)

${ }^{29}$ A. C. Sheiner, G. E. Scuseria, T. J. Lee, J. E. Rice, and H. F. Shaefer III, J. Chem. Phys. 87, 5361 (1987).

${ }^{30}$ J. Gauss, J. F. Stanton, and R. J. Bartlett, J. Chem. Phys. 95, 2623 (1991).

${ }^{31}$ H. Koch, H. J. Aa. Jensen, P. Jørgensen, T. Helgaker, G. Scuseria, and H. F. Shaefer III, J. Chem. Phys. 92, 4924 (1990).

${ }^{32}$ R. Kobayashi, K. Koch, and P. Jørgensen, Chem. Phys. Lett. 219, 30 (1994).

${ }^{33}$ H. Sekino and R. J. Bartlett, J. Chem. Phys. 98, 3022 (1993).

${ }^{34}$ H. Sekino and R. J. Bartlett, Chem. Phys. Lett. 234, 87 (1995).

${ }^{35}$ J. Gauss and J. F. Stanton, J. Chem. Phys. 102, 251 (1995).

${ }^{36}$ J. Gauss and J. F. Stanton, J. Chem. Phys. 103, 3561 (1995).

${ }^{37}$ J. Gauss and K. Ruud, Int. J. Quantum Chem. S29, 437 (1995). 
${ }^{38}$ J. Gauss and J. F. Stanton, J. Chem. Phys. 104, 2574 (1996).

${ }^{39}$ R. Kobayashi, O. Bludsky, H. Koch, and P. Jørgensen, Chem. Phys. Lett. 215, 576 (1993).

${ }^{40}$ R. Kobayashi, H. Koch, and P. Jørgensen, J. Chem. Phys. 101, 4956 (1994).

${ }^{41}$ S. Ajith Perera, M. Noijen, and R. J. Bartlett, J. Chem. Phys. 104, 3290 (1996).

${ }^{42}$ K. Raghavachari, G. W. Trucks, J. A. Pople, and M. Head-Gordon, Chem. Phys. Lett. 157, 479 (1989).

${ }^{43}$ H. Koch, O. Christiansen, P. Jørgensen, A. Sanchez de Meras, and T. Helgaker, J. Chem. Phys. (submitted).

${ }^{44}$ C. Hättig and B. A. Hess, Chem. Phys. Lett. 233, 359 (1995).

${ }^{45}$ F. Aiga, K. Sasagane, and R. Itoh, J. Chem. Phys. 99, 3779 (1993).

${ }^{46}$ H. Koch, O. Christiansen, P. Jørgensen, and J. Olsen, Chem. Phys. Lett. 244, 75 (1995).

${ }^{47}$ O. Christiansen, H. Koch, P. Jørgensen, and J. Olsen, Chem. Phys. Lett. (accepted).

${ }^{48}$ O. Christiansen, H. Koch, and P. Jørgensen, J. Chem. Phys. (submitted).

${ }^{49}$ J. D. Watts and R. J. Bartlett, Chem. Phys. Lett. 233, 81 (1995).

${ }^{50}$ J. Almlöf, K. Korsell, and K. Fægri, J. Comput. Chem. 3, 385 (1982).

${ }^{51}$ O. Christiansen, H. Koch, P. Jørgensen, and T. Helgaker, Chem. Phys. Lett. (submitted).

${ }^{52}$ P. J. Hay and I. Shavitt, Chem. Phys. Lett. 22, 33 (1973); J. Chem. Phys. 60, 2865 (1974).

${ }^{53}$ J. Maurice, O. Matos, B. O. Roos, and P.-A. Malmqvist, J. Chem. Phys. 86, 1459 (1987).

${ }^{54}$ O. Kitao and H. Nakatsuji, J. Chem. Phys. 87, 1169 (1987).

${ }^{55}$ B. O. Roos, K. Anderson, and M. Fülscher, Chem. Phys. Lett. 192, 5 (1992).

${ }^{56}$ J. Lorentzon, P.-А. Malmqvist, M. Fülscher, and B. O. Roos, Theor. Chim. Acta 91, 91 (1995).

${ }^{57}$ M. J. Packer, E. K. Dalskov, T. Enevoldsen, H. J. Aa. Jensen, and J. Oddershede, J. Chem. Phys. (submitted).

${ }^{58}$ K. Hirao, H. Nakano, and T. Hashimoto, J. Chem. Phys. 235, 430 (1995).

${ }^{59}$ E. C. da Silva, J. Gerratt, D. L. Cooper, and M. Raimondi, J. Chem. Phys. 101, 3866 (1994).

${ }^{60}$ M. H. Palmer, and I. C. Walker, J. Chem. Phys. 133, 113 (1989).

${ }^{61}$ W. C. Price and R. W. Wood, J. Chem. Phys. 3, 439 (1935).

${ }^{62}$ P. G. Wilkinson, Can. J. Phys. 34, 596 (1956).

${ }^{63}$ E. N. Lassettre, A. Skerbele, M. A. Dillon, and K. J. Ross, J. Chem. Phys. 48, 5066 (1967)

${ }^{64}$ E. E. Koch and A. Otto, Chem. Phys. Lett. 12, 476 (1972).
${ }^{65}$ P. M. Johnson, J. Chem. Phys. 64, 4143 (1976).

${ }^{66}$ P. M. Johnson and G. M. Korenowski, Chem. Phys. Lett. 97, 57 (1983).

${ }^{67}$ N. Nakashima, M. Sumiteni, I. Okmine, and K. Yoshihara, J. Chem. Phys. 72, 2226 (1980)

${ }^{68}$ N. Nakashima, H. Inoue, M. Sumiteni, and K. Yoshihara, J. Chem. Phys. 73, 5629 (1980)

${ }^{69}$ R. L. Whetten, K-J. Fu, and E. R. Grant, J. Chem. Phys. 79, 2620 (1983).

${ }^{70}$ S. G. Grubb, R. L. Whetten, A. C. Albrecht, and E. R. Grant, Chem. Phys. Lett. 108, 420 (1984).

${ }^{71}$ R. L. Whetten, S. G. Grubb, C. E. Otis, A. C. Albrecht, and E. R. Grant, J. Chem. Phys. 82, 1115 (1985).

${ }^{72}$ S. G. Grubb, C. E. Otis, R. L. Whetten, E. R. Grant, and A. C. Albrecht, J. Chem. Phys. 82, 1135 (1985).

${ }^{73}$ A. Hiraya and K. Shobatake J. Chem. Phys. 94, 7700 (1991).

${ }^{74}$ E. R. Davidson, J. Comput. Phys. 17, 87 (1975); J. Phys. A 13, L179 (1980).

${ }^{75}$ T. H. Dunning Jr., J. Chem. Phys. 90, 1007 (1989).

${ }^{76}$ R. A. Kendall, T. H. Dunning Jr., and R. J. Harrison, J. Chem. Phys. 96, 6769 (1992)

${ }^{77}$ K. Kaufmann, W. Baumeister, and M. Jungen, J. Phys. B 22, 2223 (1989).

${ }^{78}$ M. Head-Gordon, R. J. Rico, M. Oumi, and T. J. Lee, Chem. Phys. Lett. 219, 21 (1994).

${ }^{79}$ A. Staib and W. Domcke, J. Chem. Phys. 94, 5402 (1991), and references therein.

${ }^{80}$ R. L. Whetten and E. R. Grant, J. Chem. Phys. 80, 5999 (1984), and references therein.

${ }^{81}$ G. Herzberg, Electronic Spectra of Polyatomic Molecules (Van Nostrand Reinhold, New York, 1966).

${ }^{82}$ C. S. Parmenter, Adv. Chem. Phys. 22, 365 (1972).

${ }^{83}$ K. Raghavachari, R. C. Haddon, T. A. Miller, and V. E. Bondybey, J. Chem. Phys. 79, 1387 (1983).

${ }^{84}$ K. Hirao and H. Kato, Chem. Phys. Lett. 98, 340 (1983).

${ }^{85}$ V. Vaida, B. M. Robin, and N. A. Kuebler, Chem. Phys. Lett. 58, 557 (1978).

${ }^{86}$ J. Olsen, O. Christiansen, H. Koch, and O. Jørgensen, J. Chem. Phys. (submitted).

${ }^{87} \mathrm{~J}$. Olsen (private communication).

${ }^{88}$ M. Head-Gordon, J. A. Pople, and M. J. Frisch, Chem. Phys. Lett. 153, 503 (1988).

${ }^{89}$ S. Saeb $\varnothing$ and J. Almlöf, Chem. Phys. Lett. 154, 83 (1989).

${ }^{90}$ J. F. Stanton, J. Chem. Phys. 99, 8840 (1993).

${ }^{91}$ J. F. Stanton and J. Gauss, Theor. Chim. Acta 91, 267 (1995). 